\title{
High-frequency near-field microscopy
}

\author{
Björn T. Rosner \\ Department of Electrical Engineering, University of Wisconsin, Madison, Wisconsin 53706 \\ and Department of Electrical Engineering, University of Delaware, Newark, Delaware 19716 \\ Daniel W. van der Weide ${ }^{\text {a) }}$ \\ Department of Electrical Engineering, University of Wisconsin, Madison, Wisconsin 53706
}

(Received 10 October 2001; accepted for publication 7 April 2002)

\begin{abstract}
Conventional optics in the radio frequency (rf) through far-infrared (FIR) regime cannot resolve microscopic features since resolution in the far field is limited by wavelength. With the advent of near-field microscopy, rf and FIR microscopy have gained more attention because of their many applications including material characterization and integrated circuit testing. We provide a brief historical review of how near-field microscopy has developed, including a review of visible and infrared near-field microscopy in the context of our main theme, the principles and applications of near-field microscopy using millimeter to micrometer electromagnetic waves. We discuss and compare aspects of the remarkably wide range of different near-field techniques, which range from scattering type to aperture to waveguide structures. (C) 2002 American Institute of Physics.
\end{abstract}

[DOI: $10.1063 / 1.1482150]$

\section{INTRODUCTION}

Doing microscopy with electromagnetic waves implies using visible light: This is not only because our eyes can detect it, but also because its sub- $\mu \mathrm{m}$ wavelengths combined with conventional optics lead to useful resolving power. Thus, even when electronic detectors for invisible radiation became available, microscopy was not immediately extended to longer wavelengths, even though many phenomena with characteristic frequencies below the visible have been of interest. According to Abbe's much-cited criterion, ${ }^{1,2}$ resolving power is $\sim \lambda / 2$, so using millimeter wave and infrared frequencies would result in unattractively low resolution. While this criterion is correct for far-field microscopy, it is not limiting when near-field interactions are taken into account. In 1928 and 1931, 3,4 Synge proposed that super resolution could be achieved by trading the parallel data acquisition advantage of conventional optics for serial image acquisition. In his first approach, he suggested creating a subwavelength sized hole in a metal film, illuminating it from the backside and scanning it very close to the sample. While he did not refer to this intuitive idea, Bethe ${ }^{5}$ provided independent theoretical backing in his treatment of radiation emitted from a subwavelength hole in a cavity, later modified to yield the Bethe/Bouwkamp model. ${ }^{6,7}$

While both the original work of Synge and a reinvention of essentially the same concept by $\mathrm{O}^{\prime} \mathrm{Keefe}^{8}$ in 1956 were rooted in the visible regime, the first practical implementation of near-field microscopy was developed for rf applications, a localized magnetic field microscope. Frait ${ }^{9}$ (1959)

${ }^{a)}$ Electronic mail: danvdw@engr.wisc.edu and Soohoo ${ }^{10}$ (1962) independently developed a microwave cavity with a small hole that, when scanned close to a surface, would be sensitive to local changes in the magnetic properties of the sample. In analogy to the development of the laser from the maser, the experimental realization of electromagnetic near-field imaging was first accomplished in the microwave regime because the dimensional requirements for probe size and distance to the sample were much less stringent.

The first use of a coaxial waveguiding probe to localize microwave fields for measuring material properties dates back to 1965 when Bryant and Gunn ${ }^{11}$ employed a tapered coaxial tip to measure local resistivity of semiconductor samples at $450 \mathrm{MHz}$ with millimeter resolution. While this publication seems to be the first that presents deep subwavelength resolution, it was a contribution by Ash and Nicholls ${ }^{12}$ in 1972 that had the most profound influence on the development of modern near-field instruments. They demonstrated $\lambda / 60$ wavelength-relative resolution using $3 \mathrm{~cm}$ microwaves confined to a subwavelength aperture. An aperture-based approach was also the first to be employed in the visible regime.

Before near-field microscopy could be developed to any higher degree of resolution, a few technical difficulties had to be overcome. First, the near-field probe must be scanned over the sample at a distance less than the resolution sought. Since highly confined electromagnetic fields are sensitive to variations in the probe-sample separation, ${ }^{13}$ variations in this critical parameter often lead to topographical artifacts in the near-field image. Thus, probe-sample gap control in the nanometer range and advanced vibration isolation techniques are critical. The advent of scanning tunneling microscopy 
$(\mathrm{STM})^{14}$ and subsequent scanning probe techniques provided a means by which such tight distance control became possible. Consequently, after initial investigation of subwavelength sized holes ${ }^{15,16}$ in flat opaque films, the first scanning near-field optical microscopes (SNOM) were developed by Pohl et al. ${ }^{17,18}$ and shortly thereafter by Betzig et al. ${ }^{19}$ using STM feedback for distance control. At about the same time, in the mid 1980s, Massey reported on proof-of-principle experiments for FIR near-field microscopy, ${ }^{20}$ also based on the aperture technique, while seminal work demonstrating microwave subwavelength field resolution with a coaxial tip was done by Fee, Chu, and Hänsch ${ }^{21}$ in 1989 , albeit without tip-sample distance control. Meanwhile, in the visible regime, researchers were looking for an alternative to STM that would allow scanning of insulating samples because nonconducting materials could not be imaged using STM until the development of alternating current STM (AC-STM) in 1989 by Kochanski. ${ }^{22}$ Thus the advent of scanning force microscopy (SFM) in $1986^{23}$ was followed by SNOM instruments relying on (shear-) force feedback ${ }^{24,25}$ in 1992. From that point, near-field microscopy has spread through a wealth of techniques and applications to form a diverse and very active field of research.

In this review, we will briefly outline major developments in SNOM and scanning near- field infrared microscopy (SNIM) before we move on to the main topic of this article, SNIM in the FIR and scanning near-field microwave microscopy, which we will abbreviate as SNMM. This review is not meant as a complete guide to near-field microscopy encompassing experimental and theoretical work alike. Rather it is intended to organize and compare techniques and designs in SNMM while emphasizing applications where appropriate. While in the visible regime much theoretical understanding has been won, a review of theoretical concepts is outside the scope of this review. For near-field microscopy in the microwave regime, there is little theoretical work focusing on the particular designs presented. Issues like artifacts, perturbations due to the probe, measured components of the microwave signals and deconvolution of images have not yet received much attention. We believe that as the field of microwave near-field microscopy grows, these voids will be filled in time.

\section{SCANNING NEAR-FIELD OPTICAL MICROSCOPY}

To provide the context in which SNMM has been developed, let us briefly review scanned probe optical techniques for microscopy. Initial efforts in SNOM were focused on producing a nanometer sized opening to be used for transmission near-field microscopy. The first SNOM instruments used etched quartz crystals ${ }^{17,18}$ or micropipettes, ${ }^{19,26,27}$ sharpened and coated with an opaque metallic layer that exposed an aperture smaller than $100 \mathrm{~nm}$ at the apex (Fig. 1). Today, the most common probes are sharpened optical fibers and several commercial products based on fiber SNOM are now available. The original STM feedback has been replaced by shear-force feedback, ${ }^{24,25}$ and the tedious optical detection of the shear-force signal has largely been replaced by quartz tuning fork feedback ${ }^{28,29}$ or similar techniques. One major

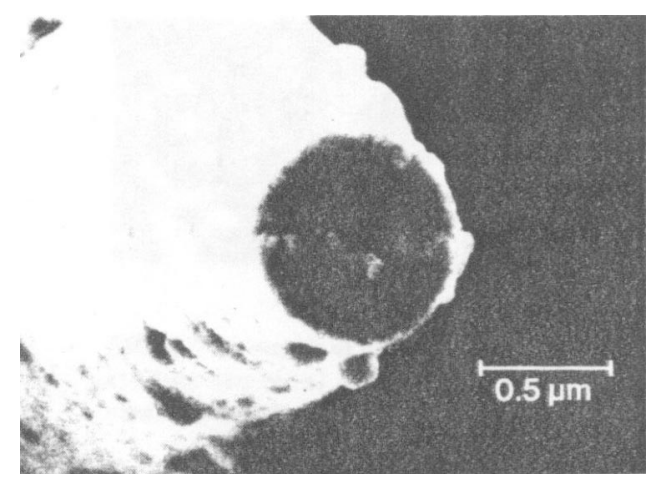

FIG. 1. Scanning electron microscopy (SEM) micrograph of an early aluminum coated pipette SNOM probe (see Ref. 27).

concern for optical near-field microscopy is the low signal strength, in this case the low transmission through SNOM fibers since they act as cutoff waveguides for light near the apex. To improve transmission over that of the common technique of pulling heated fibers, meniscus etching (the Turner method) has been employed. ${ }^{30}$ For even higher throughput, double-tapered probes have been used. ${ }^{31}$ Tube etching, another etching technique where the polymer coating of the fiber is not removed prior to etching, has been introduced $^{32}$ and hybrid techniques that use both pulling and etching for better throughput have been reported. ${ }^{33,34}$ For further information regarding aperture SNOM, there are two excellent reviews of the field by Hecht et al. ${ }^{35}$ and Dunn. ${ }^{36}$

As a complement to aperture SNOM, apertureless SNOM has been introduced, with the usage of uncoated fibers in collection mode lying on the boundary between these two families of techniques. Apertureless SNOM includes a wide variety of related approaches, including photon tunneling, use of surface plasmons and scattering-type SNOM (s-SNOM).

While making use of plasmon-guiding effects to localize light is still in its infancy, considerable interest in this field exists ${ }^{37-42}$ and the concept of using a tetrahedral tip with an integrated plasmon guide as a scanning probe has been explored $^{43-45}$ (Fig. 2). This technique has significant potential for improving SNOM because it can increase the light

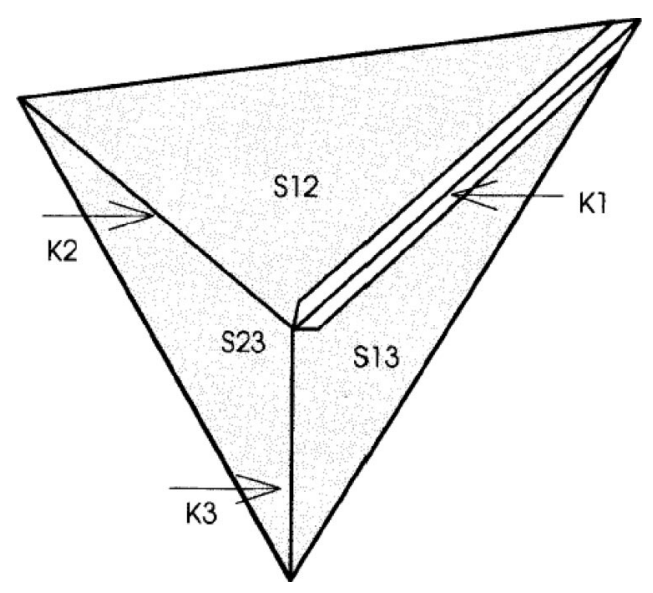

FIG. 2. Tetrahedral tip for plasmon-assisted SNOM. All of the faces and edges are coated with gold except the edge $\mathrm{K} 1$, which is coated with less gold due to the oblique evaporation process (see Ref. 43). 
a)

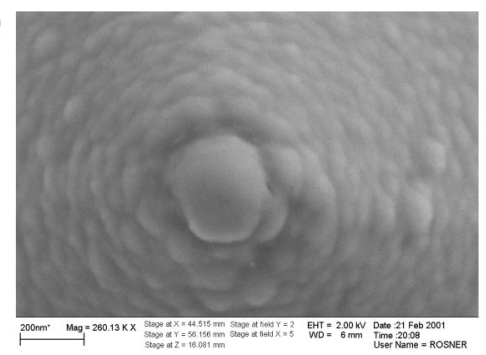

b)

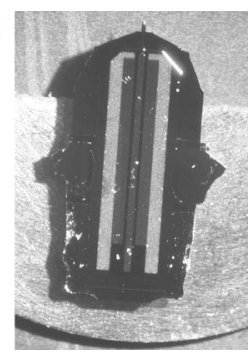

FIG. 3. (a) SEM micrograph of a nanometer-scale Schottky diode at the apex of a SFM tip. (b) This SNOM detector is integrated on a cantilever and chip body that contains waveguides for straightforward connection to macroscopic instrumentation.

throughput by orders of magnitude, as is seen in surface enhanced Raman scattering (SERS). ${ }^{46}$

The s-SNOM technique has been introduced in recent years. ${ }^{38,47-50}$ It will be examined in more detail when we discuss infrared (IR), FIR and millimeter wave microscopy because its application and resolution is wavelength independent in contrast to aperture SNOM, which requires a waveguide. The optical contrast of s-SNOM arises from the interaction between a sharp tip (for example, a STM or SFM tip) and the sample, leading to characteristic local scattering. The main advantage of this technique is that the resolution is not limited by an aperture or the skin depth of metal, but rather by the sharpness of the probing tip, potentially leading to better resolution. The drawback of s-SNOM is that a large amount of background radiation impinges on the sample and must be distinguished from the tip-sample scattering signal. Furthermore, the exact source of contrast and the implications thereof are still matters of debate. Nonetheless, both areas have been addressed in recent publications ${ }^{51-54}$ and are discussed in detail below.

Finally, researchers have been trying to microfabricate SNOM probes that could be applied in the same fashion as cantilevered SFM probes, with minor instrumental changes. Some of the proposed concepts are passive, like metal-coated probes with slit-shaped ${ }^{55}$ or circular ${ }^{56-62}$ apertures (reminiscent of fiber SNOM tips) and solid immersion lenses on cantilevers $^{63-66}$ (strictly speaking a far-field technique).

Initial efforts have been made to fabricate active devices in GaAs that include integrated light sources ${ }^{67,68}$ Considerably more research has been done to produce photodetectors integrated close to the probe ${ }^{69-72}$ or right at the apex of the sharp tip ${ }^{73-75}$ preferably integrated with the cantilever and chip body ${ }^{76,77}$ Our group has recently developed this type of cantilever-integrated photodetector SNOM, shown in Fig. 3, with fully integrated electrical connections and adapters to macroscopic instrumentation. ${ }^{78,79}$

\section{SCANNING NEAR-FIELD INFRARED MICROSCOPY}

Scanning near-field microscopy in the infrared got its main impetus from traditional bulk techniques employed by chemical spectroscopists. Because of the wealth of chemical information available through vibrational band spectroscopy, many traditional techniques like Fourier transform infrared spectroscopy (FTIR), thermal probing and Raman scattering have become cornerstones of chemical characterization. Ra-
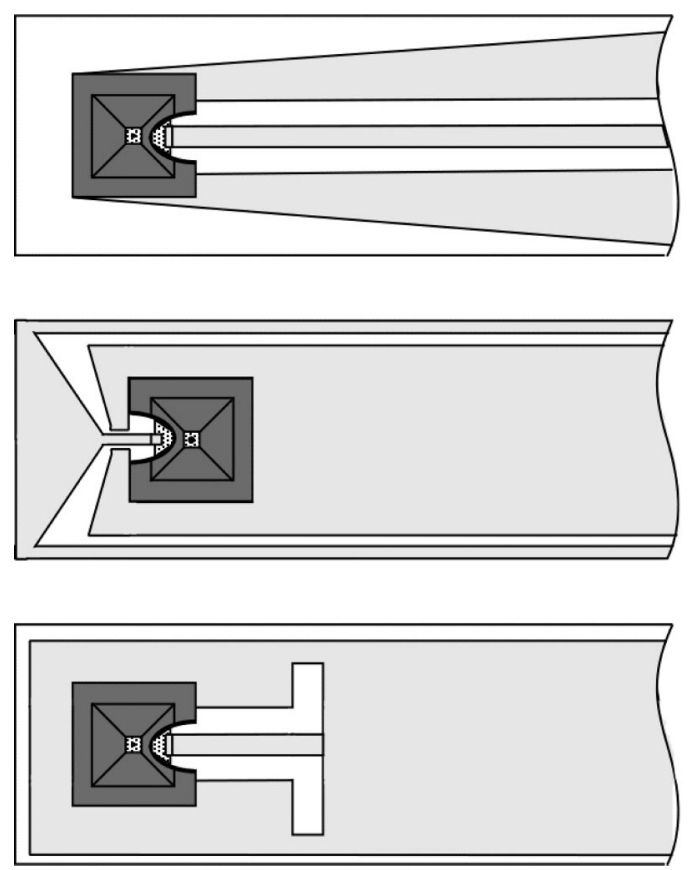

FIG. 4. Scanning probe microscopy (SPM) cantilevers incorporating waveguides and antennas feeding near-zone coaxial tips. From top to bottom: Coplanar waveguide, slot-V antenna, resonant slot antenna (see Ref. 101).

man scattering takes a unique place in this list since it is strictly speaking a SNOM technique - the light used is visible, but the energy shifts correspond to infrared wavelengths. Proximal probes offer a way to combine these techniques with subwavelength resolution. Since the wavelengths involved are an order of magnitude larger than those in visible microscopy using the same type of probe fabrication, the gain in resolution compared to far-field techniques is larger, making near-field measurements even more desirable. On the downside, there are major technical difficulties to be overcome. Very bright and tunable light sources are necessary to overcome the relatively low sensitivity of IR detectors compared to avalanche photodiodes and photomultiplier tubes used in the visible spectrum. Furthermore, the transition from far field to near field is usually less efficient due to the longer wavelength, although this depends on the particular technique. Finally, the cross sections of vibrational transitions are also often smaller than those of electronic transitions accessed in the visible regime.

Photon scanning tunneling microscopy (also called scanning tunneling optical microscopy) and related total internal reflection (TIR) setups ${ }^{80-83}$ were the first employed for nearfield scans in the IR. Fiber based aperture SNIM setups have been developed, based on special infrared transmissive fibers and etching techniques. ${ }^{84-92}$ Just as in the visible spectrum, s-SNOM has been applied with very good wavelengthrelative resolution. ${ }^{54,93-95}$ Other techniques include using transient photoinduced near-field probes ${ }^{96}$ and the use of resistive thermal probes for localized FTIR microscopy. ${ }^{97}$ A promising approach could be the use of antenna structures (Fig. 4) as near-field sources. ${ }^{98-101}$ While any coaxial openended waveguide can be considered an antenna, guiding radiation at visible or infrared wavelengths is not yet techno- 
logically feasible. However, by using antennas that collect far-field radiation and channel it to near-field tips for exciting the sample (or vice versa, see Fig. 4), antenna concepts can be very effective. While any metallic particle or s-SNOM tip is in principle an antenna, the efficiency of such structures can possibly be improved by applying engineering design rules currently used in microwave engineering. Even in the 1970's, discrete devices that employed small gaps between conductors and acted as antennas were investigated. Both point-contact "cat whisker" antennas ${ }^{102-105}$ and metalbarrier-metal diodes ${ }^{106}$ were used to rectify or mix incident far-field FIR or IR radiation. Closely related are mixing experiments in a STM junction ${ }^{107-109}$ that are discussed in detail in Sec. VA3. The performance of such discrete devices agreed fairly well with theoretical predictions from antenna theory. ${ }^{103,105}$ Although application of antennas to the visible regime is possible in principle, we have found that scaling engineered antennas to visible frequencies is not straightforward because of material nonidealities and fabrication difficulties. ${ }^{101}$ For example, surface roughness and oxidation become important loss factors at these scales. If semiconductor fabrication techniques are employed to produce submicron features, substantial substrate losses are limiting factors because the substrate cannot be made thin enough. At infrared wavelengths, however, lithographically defined antennas have already been successfully applied to collect far-field radiation. ${ }^{105,110-113}$ Fabrication proposals for IR and FIR antenna near-field probes exist ${ }^{101}$ and optical antenna design was recently discussed by Pohl. ${ }^{100} \mathrm{We}$ expect a growing interest in engineered antennas for infrared to visible frequencies in the near future. Excellent reviews of nearfield infrared microscopy in general and near-field vibrational spectroscopy have been written by Dragnea and Leone ${ }^{114}$ and by Pollock and Smith. ${ }^{115}$

\section{SCANNING NEAR-FIELD MICROSCOPY IN THE FAR INFRARED}

Although many electromagnetic phenomena with characteristic frequencies below the near infrared but above the radio bands are of interest, surprisingly little work has been done on near-field microscopy in the far infrared, which we define as radiation $10 \mu \mathrm{m}<\lambda<1 \mathrm{~mm}$ (corresponding to 300 $\mathrm{GHz}$ ). These phenomena include biological membrane absorption and conductive or dielectric properties of materials, e.g., superconductors and quantum dots. The $\sim 10 \mu \mathrm{m}$ range of FIR is also part of the molecular fingerprint regime because transmission or reflection experiments in the FIR reveal more chemical information than in any other frequency band. Thus, FIR localized microscopy is highly desirable for mapping molecular rotational or vibrational absorption spectra, i.e., chemical microscopy. The lack of research in this area arises from inherent difficulties in dealing with farinfrared radiation. While there are reliable and intense sources of radiation at the short-wavelength end of FIR (e.g., $\mathrm{CO}_{2}$ lasers emitting at $10.6 \mu \mathrm{m}$ ), the same cannot be said for wavelengths $>100 \mu \mathrm{m}$, especially since tunability is necessary to record spectra. To get bright FIR sources, many researchers use bulky and maintenance intensive gas lasers,

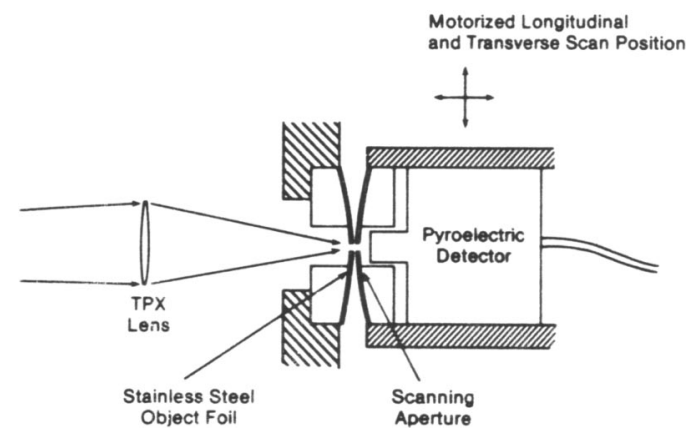

FIG. 5. A schematic of the scanning aperture employed in the first FIR near-field experiment conducted by Massey (see Ref. 20).

pumped with a $\mathrm{CO}_{2}$ laser. Other approaches for FIR sources include $\mathrm{THz}$ time domain spectroscopy using photoconductive $\mathrm{THz}$ emitters ${ }^{116}$ pumped by femtosecond optical lasers and all-electronic generation of $\mathrm{THz}$ radiation with nonlinear transmission lines (NLTL). ${ }^{117-119}$. The invention and continuing development of quantum cascade lasers ${ }^{120-124}$ has fueled hopes that a compact, tunable and high-power FIR source for long wavelengths will be commercially available in the near future. Another problem, however, is sensitive FIR detection. Situated between the visible regime, where optoelectronic properties of semiconductors can be used for detection, and the microwave region, where direct electronic detection of amplitude and phase is possible with vector network analyzers, FIR detection has to rely on power detection via Schottky diodes, which are often fragile, or bolometers, which are costly and slow. If these obstacles can be overcome, near-field imaging with far-infrared light will be very attractive. We now discuss the different approaches taken for FIR SNIM.

\section{A. Aperture FIR-SNIM}

The first work on near-field microscopy in the FIR was done by Massey et al. ${ }^{20}$ in 1985, which followed initial scale modeling at $450 \mathrm{MHz}$ in $1984 .{ }^{125}$ Their proof-of-principle experiment consisted of two $10 \mu \mathrm{m}$ pinholes being scanned against each other as shown in Fig. 5. Massey et al. used a $118.8 \mu \mathrm{m}$ methanol vapor laser, demonstrating resolution down to $30 \mu \mathrm{m}$. It was quickly realized that it would be practical to extrude the aperture in a flat sheet to form a three-dimensional (3D) funnel or tapered cylindrical waveguide, and this geometry was first employed by Keilmann and Merz ${ }^{126,127}$ to overcome the imperfect focusing capabilities of traditional FIR optics. As pointed out by Keilmann, ${ }^{128}$ power transmittance in the cutoff region is

$$
T=e^{-4 \pi \sqrt{\left(f_{c}^{2}-f^{2}\right)} \cdot t},
$$

where $t$ is the penetration depth into the cutoff region, $f$ and $f_{c}$ are the frequency of the radiation and the cutoff frequency, respectively. In practice, they found a sharp cutoff in transmitted intensity at an aperture size of about $\lambda / 2$. They then applied this system for far-infrared near-field spectroscopy of two-dimensional (2D) electron systems. Aperture confinement of FIR radiation has been one of the leading techniques to date ${ }^{116,128}$ because of its straightforward implementation. 


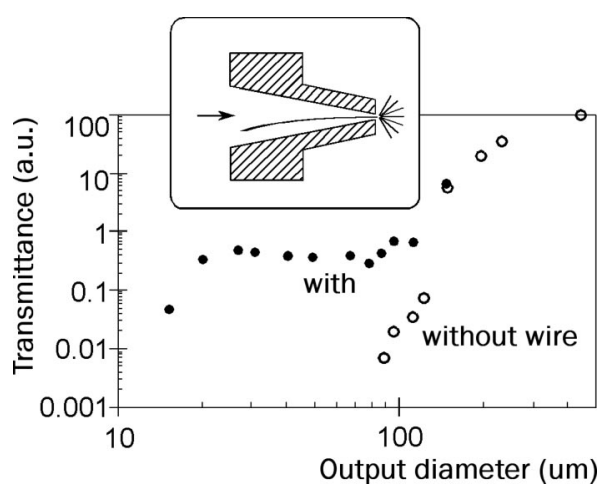

FIG. 6. The relative transmittance at a wavelength of $\lambda=392 \mu \mathrm{m}$ of focusing cones with different output apertures is investigated. Adding a central, coaxial wire prevents cutoff reflection at $d<\lambda / 2$ (see Ref. 128).

\section{B. Coaxial waveguide FIR-SNIM}

Unlike a hollow metal waveguide, a guide with two conductors, if appropriately chosen, can guide radiation without cutoff. This means that the waveguide can be tapered down in its lateral dimension to a size much smaller than the wavelength of the guided wave without losing much of the incident intensity to reflection, scattering and absorption. It is well known from electromagnetic theory that circular and rectangular hollow waveguides have a cutoff wavelength roughly corresponding to double their inner diameter, while a coaxial waveguide has no cutoff at all. Therefore, it was proposed early on ${ }^{21,129-131}$ that coaxial waveguides would be the ideal way to confine incident visible light without losses. Although application of this principle to visible light has not yet been realized, due to the material and fabrication challenges involved, coaxial waveguides have many applications in near-field microscopy in the microwave regime, and they were investigated by Keilmann ${ }^{126,128}$ in the FIR. Keilmann et al. showed that by introducing a center conductor into the tapered hollow guide used before, they could decrease the aperture size by almost an order of magnitude without losing transmitted power (Fig. 6). They also found that the transmitted power varied with a period of $\lambda / 2$ as they extended the length of wire protruding from the aperture; it acts as a resonant antenna.

\section{Scattering FIR-SNIM}

Using apertureless (scattering) SNIM in the FIR has even better prospects than in the visible regime. For one, since the resolution depends on the scattering tip, not the wavelength, the wavelength-specific resolution is far better. FIR microscopy with nanometer resolution is possible and has been demonstrated. ${ }^{54,93-95,132}$ (Fig. 7). Furthermore, since the wavelength is much longer than the occasional distance variations between tip and sample, field interference effects play less of a role, reducing the risk of topographical artifacts in the optical data.

While the image contrast mechanism of aperture-based near-field microscopy is understood in its basics, ${ }^{133,134}$ the field is still wide open to investigation for apertureless scattering-type near-field microscopes. Following one argument, ${ }^{52-54}$ image contrast arises from the interaction be-

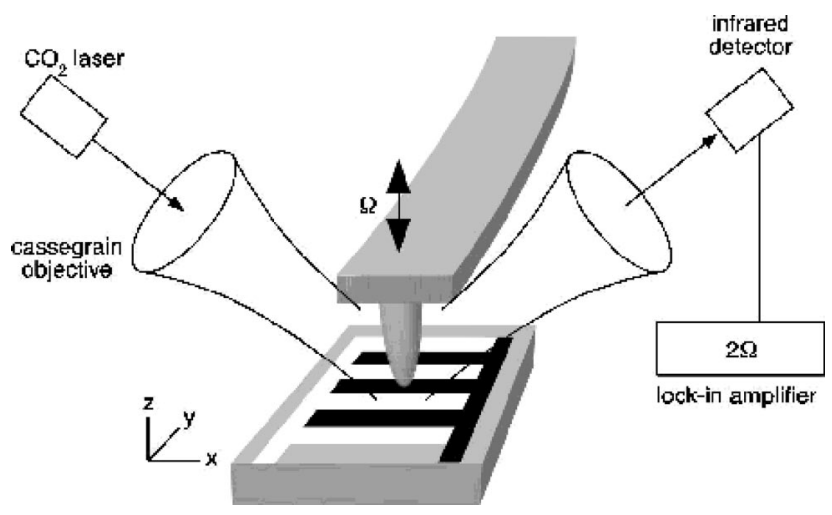

FIG. 7. Sketch of a scattering-type near-field optical microscope. The scattering tip is illuminated by a focused beam and the scattered light is collected (see Ref. 132). Here, higher order modulation is used for background suppression.

tween the polarizable probe (modeled as a sphere with polarizability $\alpha$ and radius $a$ ) and the dielectric or metallic sample with complex dielectric constant $\epsilon$. This results in an effective polarizability of the coupled system

$$
\alpha_{\perp}^{\mathrm{eff}}=\frac{\alpha(1+\beta)}{1-\frac{\alpha \beta}{16 \pi(z+a)^{3}}},
$$

where $\beta=(\epsilon-1) /(\epsilon+1)$ and $z$ is the probe-sample separation. This formula shows both strong enhancement at short distances and the existence of amplitude and phase effects, since $\alpha$ and $\beta$ are complex. The scattered far-field $E_{\text {sca }}$ $=\alpha^{\text {eff }} E$ thus reports the phase and amplitude of the complex near-field interaction. ${ }^{53}$ Further discussion of theoretical work done on s-SNOM has appeared recently. ${ }^{54,135-140}$

\section{Other techniques for FIR-SNIM}

While aperture probes, scattering probes and coaxial tips form the main body of the research done so far, some researchers have proposed independent ideas that do not fit readily into these categories.

Mitrofanov et al. ${ }^{141}$ have built a hybrid proximal probe, which is based on a photoconducting antenna connected to a GaAs taper with subwavelength aperture. With an ultrashort optical pulse incident on the antenna through a sapphire substrate, the $\mathrm{THz}$ radiation created in the photoconductive antenna is coupled into the adjacent metal-coated GaAs taper with aperture at its apex. The near-field probe exhibits a broadband spectrum in the range of $0.3-1.5 \mathrm{THz}$; spatial resolution is $50 \mu \mathrm{m}$.

Earlier work by Nakano and Kawata ${ }^{83}$ employs a TIR setup where the total internal reflection does not occur on the sample side, but inside a $\mathrm{ZnSe}$ tip that is used for scanning.

Reflection measurements with transient photoinduced reflectivity achieve subwavelength resolution in the FIR through a novel approach. ${ }^{96}$ A pulse of visible light is absorbed on the sample and generates a transient electron-hole plasma that shows high reflectivity $(>70 \%)$ for $10.6 \mu \mathrm{m}$ 
radiation. By illuminating this spot with infrared light, only the much smaller optically illuminated area reflects the beam.

An entirely different approach to FIR microscopy lies in the use of photothermal signals for near-field spectroscopy. Here, a thermal probe in temperature-sensing mode directly measures absorption of incident radiation by sensing the induced heating of the sample. This heating can either be directly measured for each wavelength in the dispersive approach $^{142}$ or a Fourier transform absorption spectrum can be acquired when using intensity-modulated illumination. ${ }^{97}$

The brevity of this part of the review, near-field microscopy with FIR radiation, indicates the magnitude of the technical challenges of FIR imaging, particularly with sources and detectors. With the advent of compact, intense FIR sources, a significant increase in research activity in this area can be expected.

\section{SCANNING NEAR-FIELD MICROWAVE MICROSCOPY}

Localized measurements at microwave frequencies are primarily motivated by the need for characterization and quality assurance of materials and integrated circuits (ICs), where far-field scanned beam techniques like electron beam testing, ${ }^{143}$ photoemission sampling ${ }^{144}$ and electro-optic sampling ${ }^{145-147}$ are commonly employed. We will focus on scanned probes, discussing techniques that use microwaves for illumination and detection of sample properties. We will also briefly mention some other related techniques that could be or are being used in material and IC characterization.

Microwave near-field probes are broadband or resonant. Broadband probes typically consist only of a (transverse electromagnetic) waveguide, while resonant structures employ a cavity that is coupled to the substrate through a subwavelength-sized probe. Resonant probes are more sensitive, but have to be operated in a narrow frequency range. While these two concepts overlap considerably, they offer a useful way to categorize the wide range of probes devised.

\section{A. Broadband SNMM}

Near-field microscopy in the microwave regime is the oldest of all near-field disciplines because subwavelength resolution can be achieved quite easily, and topography cross talk is much less of a problem with long wavelengths than with the visible. As mentioned in the introduction, initial developments by Frait, ${ }^{9,148}$ Soohoo $^{10}$ as well as Ash and Nicholls ${ }^{12}$ applied the aperture approach to microwave microscopy. Decréton and Gardiol ${ }^{149}$ introduced the idea of using a flanged rectangular waveguide for localized measurements of complex permittivity.

Aperture probes and tapered hollow waveguides ${ }^{150}$ have the inherent disadvantage that the incident radiation will have to pass a cutoff region, which reduces throughput considerably. For this reason, aperture (iris) near-field microscopes in any frequency regime use an aperture $>\lambda / 20$. While this might still be appropriate for optical microscopy, employing iris probes in the microwave regime would limit the maximum resolution to the millimeter range while still

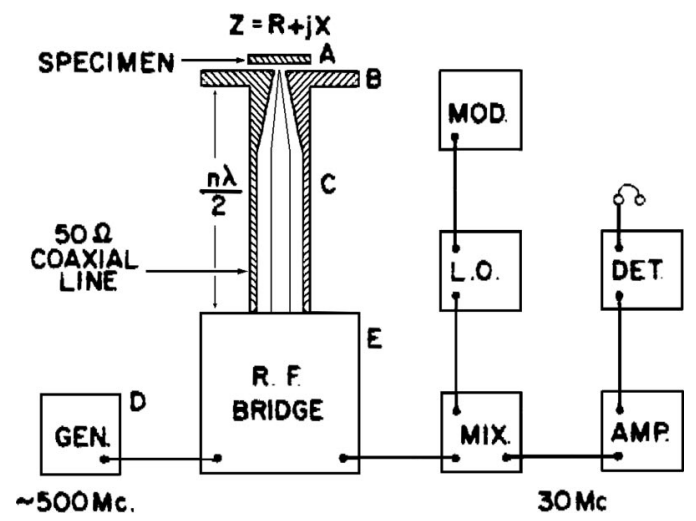

FIG. 8. The original measurement setup of Bryant and Gunn (see Ref. 11). The coaxial tip is tapered, maintaining a $50 \Omega$ characteristic impedance.

losing considerable power. Other techniques offer nearly lossless transmission and higher resolution.

\section{Macroscale SNMM}

Bryant and Gunn ${ }^{11}$ were the first to employ a tapered noncutoff waveguide, a coaxial tip as shown in Fig. 8. Coaxial waveguides have a twofold advantage in that there is no cutoff wavelength limit and that the outer conductor shields the signal, reducing noise levels significantly. Thus, flanged or flange-less open-ended coaxial waveguides have been studied in theory ${ }^{151-160}$ and used for semiconductor, superconductor and dielectric characterization, ${ }^{153,154,161,162}$ IC probing ${ }^{163}$ and even quality assurance of processed food. ${ }^{164}$ The resolution of these systems without tip- sample distance control is in the millimeter to centimeter range.

Gao and Wolff have proposed and fabricated an electric field sensor that consists of a coaxial waveguide with a dipole antenna at its end to measure $3 \mathrm{D}$ fields. ${ }^{165}$ They also created a miniature magnetic probe, consisting of a wire loop, $700 \mu \mathrm{m}$ in diameter ${ }^{166}$ while Osofsky and Schwartz designed a magnetic field probe consisting of a double loop. ${ }^{167}$ Kanda devised and theoretically analyzed an electromagnetic near-field sensor for simultaneous electric and magnetic field measurements. ${ }^{168}$

\section{Coaxial tips integrated with scanning force microscopy}

In order to increase resolution from centimeters to micrometers or less, two principal aspects of these probes must be improved. First, the probe has to be very close to the surface at all times, which can be accomplished by using force or tunnel current feedback. The resolution of the probe itself also has to be improved, which can be done by tapering the waveguide to reduce the probe opening or by sharpening the end of the inner conductor, as pointed out by Fee, Chu, and Hänsch. ${ }^{21}$ This helps concentrate the fringing fields emanating from the probe apex (lightning rod effect) and thus confines the interaction area between the probe and sample. Proof-of-principle experiments with a sample between two such sharpened coaxial transmission line tips by Keilmann et al. ${ }^{50}$ resulted in $\lambda / 10^{6}$ wavelength-relative resolution.

Integrating coaxial structures with cantilevered SFMs enables environmental (i.e., room temperature and pressure) 


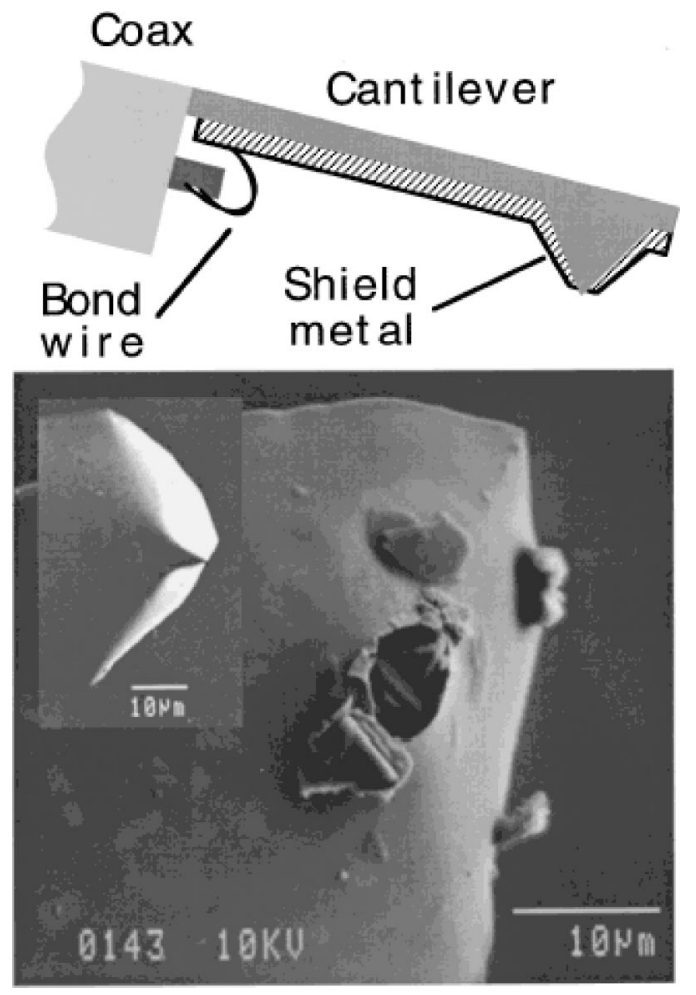

FIG. 9. Schematic arrangement of shielded SFM tip and cantilever, connected to a coaxial output cable (above), and SEM image of actual probe surrounded by coaxial shield (below). The inset shows the same tip before mechanically removing the shield (see Ref. 169).

probing of passive or active samples. Typically, the microwave signal is incident onto the SFM tip/cantilever and reflected back into a sensor at the tip-sample interface, but measurements in transmission mode are also possible. Sample properties change the phase and amplitude of the reflected signal in a way characteristic of the local dielectric or electric field.

Initial probes from our group ${ }^{169}$ were made by coating a commercial silicon SFM probe with photoresist and gold, then opening the coaxial structure by rubbing the tip gently on a substrate while in feedback (Fig. 9). While we have been able to locally measure wave forms on a fast IC, this approach has multiple drawbacks: This is not a batch process and opening the outer metal layer as described above is not reliable and reproducible. Furthermore, the double layer of metal does not act as a good waveguide for higher frequencies. Another problem, addressed by Heisig and Oesterschulze, is that $\mathrm{Si}$ is a rather lossy substrate at microwave frequencies. They show tip fabrication on GaAs and report superior signal transfer. ${ }^{67,170,171}$

Nonetheless, most work done in microfabrication of coaxial conductors is done in silicon. ${ }^{60}$ One major problem with multifunctional SFM probes is that the signal needs to travel along the cantilever and chip body in order to be collected at the far side from the tip. Since the metallization is on the sample side, care has to be taken to design the SFM chip and connecting instrumentation so that the electrical connection does not interfere with the tip scanning along the surface. To address this, we have designed and fabricated a microscopic coaxial tip together with a coplanar waveguide a)

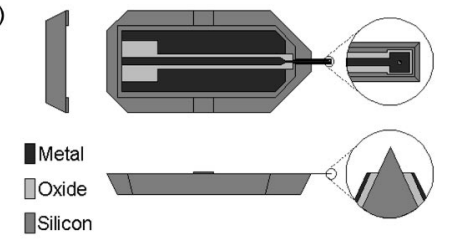

b)

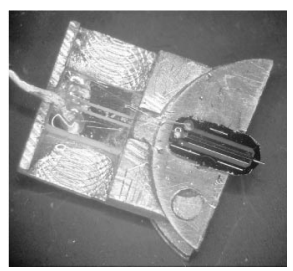

FIG. 10. (a) Schematic of coaxial tip, cantilever with microstrip and chip body integrated with CPW. (b) Probe interface to macroscopic instrumentation: The chip body connects to a waveguide transition with gold wires. The transition is permanently connected to a very thin coaxial cable and SMA connector.

(CPW) along the chip body and an interface to easily and reliably make electrical connections between the probe and external instrumentation ${ }^{79}$ as shown in Fig. 10.

\section{Coaxial tips integrated with scanning tunneling microscopy and nonlinear interactions}

Just as with SFM, STM can offer a tip-sample distance feedback system for microwave near-field microscopy while providing a sharp conducting tip to localize high-frequency electromagnetic interaction. One such system was developed by Kramer et al. ${ }^{172}$ The sample consisted of a conducting $\mathrm{Si}$ slab covered with a metal layer of varying thickness at the front. Direct current (dc) tunnel current was established between this metal layer and the scanning tip while microwaves were directed at the backside of the Si sample. These were collected through the STM tip and shielding on the sample front side. Since the tip was used in collection rather than emission mode, the microwave field strength at the tip was minimized, such that rectification and harmonic generation at the tunneling junction did not play a discernable role.

This technique, using direct current STM and microwave collection through the tip, stands in contrast to techniques based on AC-STM invented by Kochanski. ${ }^{22}$ AC-STM was first used as a means to image nonconducting surfaces. Since electrons tunnel to and from the surface as the alternating current changes polarity, no net charge transfer to the sample occurs, removing the primary restriction of scanning only conductive samples. Because field concentration is strong at the tip if the microwave signal comes from the STM side and because of tunnel junction nonlinearities, higher harmonic signals are generated and were initially used as the source for feedback, ${ }^{173}$ allowing for atomic resolution. Since the higher- harmonic generation strongly depends on the electronic properties of the surface, it is possible to differentiate different chemical species. Weiss et al. ${ }^{174-181}$ and Michel ${ }^{173}$ have explored this possibility in a number of publications. Weiss et al. have demonstrated AC-STM in environmental conditions $^{174}$ and at low temperature and ultrahigh vacuum. ${ }^{177}$ The coaxial shielding was typically extended as close to the tunneling tip as possible to reduce noise levels. While for some configurations no cavity was used, others incorporated a miniature cavity to surround and shield the sample and tip. Here, the continuous electrical connection between scanner and sample was realized using a liquid metal seal. ${ }^{179}$ These AC-STMs have been applied from dc to $20 \mathrm{GHz}$, recording linear or nonlinear (higher-harmonic) ${ }^{178}$ 


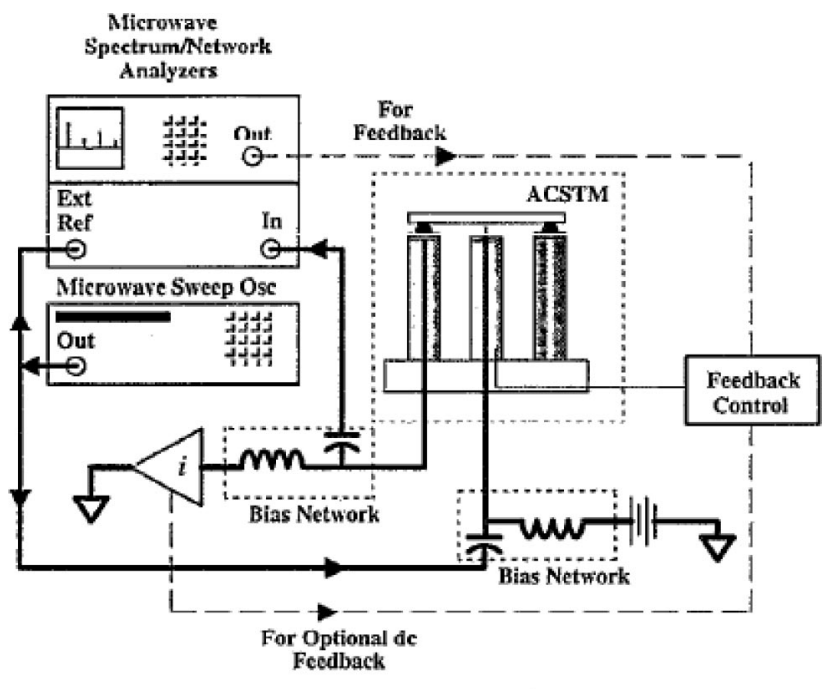

FIG. 11. Schematic of the AC-STM developed by Stranick and Weiss (see Ref. 175). Feedback dc components are separated from ac spectroscopy components by bias networks.

microwave spectra. Usually, topography has been controlled using conventional STM while simultaneously recording millimeter wave amplitude in transmission or reflection mode (Fig. 11).

These nonlinear effects between a conducting tip and a close by sample have also been exploited for other applications. Frequency mixing of two incident IR beams at a metal-metal point-contact diode and detection of a microwave difference frequency were shown as far back as 1969. ${ }^{102}$. Völcker et al. ${ }^{107-109}$ have investigated frequency mixing of two IR lasers at the junction of a STM and found the resulting rectified current and difference frequency signal at $9 \mathrm{GHz}$ dependent on sample conductivity. In a related experiment, Bragas, Land, and Martínez ${ }^{182}$ determined the optical field enhancement in a STM junction by measuring the rectified current. The researchers found an enhancement factor between 300 and 2000, partially depending on the sample underneath the STM tip. Zayats and Sandoghdar ${ }^{183}$ proposed to use the second harmonic generated at the tipsample junction as a source of contrast for s-SNOM. They found numerical justification that the field confinement for the second harmonic should be better than for the fundamental (incident) radiation, providing better spatial resolution. Furthermore, the strong background radiation at the fundamental could be ignored by detecting only the second harmonic. Related schemes to record pure optical contrast in scattering-type near-field microscopy with significantly reduced background noise and topography artifacts were introduced by Labardi, Patane, and Allegrini ${ }^{51}$ and Hillenbrand, Keilmann, and Knoll. ${ }^{53,54}$ Here, when applying noncontact SFM, the nonlinear dipole-dipole interaction between the tip and sample leads to higher modulation harmonics in the scattered signal, which can be used for lock-in detection.

\section{Scanning capacitance microscopy (SCM)}

Scanning capacitance microscopy (SCM) is not of necessity a high-frequency technique, and it is an active enough area of research to fill a review article on it own.
Nonetheless, the high-frequency capabilities of some configurations and the proximity to the technique of AC-STM justify a short review of SCM in this context. In fact, nonlinear AC-STM was used by Bourgoin, Johnson, and Michel $^{184,185}$ to measure dopant profiles in semiconductors. Siefert et al. ${ }^{186}$ report that the second and third harmonic signals are proportional to the first and second derivatives of the tip-sample capacitance. Traditionally, SCMs directly measure the capacitance between the scanning tip and the sample using a capacitance sensor. By applying a bias to the sample, $C-V$ curves can be recorded and converted to doping profiles. Scans are either performed in constant capacitance mode, ${ }^{187}$ in a SFM setup in contact ${ }^{188}$ or noncontact $^{189}$ mode or a hybrid mode which regulated the bias while keeping distance and capacitance constant. ${ }^{190}$ These systems typically have resolutions $>100 \mathrm{~nm}$ and are not designed for high-frequency operation. Lányi, Török, and Rehurek ${ }^{191-193}$ presented a SCM that achieved high resolution (10 nm laterally) and an operating frequency up to $5 \mathrm{MHz}$. By employing a coaxial probe, they found that stray capacitance could be reduced by orders of magnitude, resulting in better signal-tonoise ratio and thus higher frequency operation as well as better resolution. SCM has also been employed simultaneously with SNOM imaging using bent gold-coated fibers to localize gate-oxide leakage in polished static random access memory integrated circuits. ${ }^{194}$

\section{Scattering from a conducting tip}

Scattering of incident far-field microwave radiation from a conducting tip in close proximity to a sample is analogous to s-SNOM in the visible, IR or FIR. Thus numerical and theoretical treatments of s-SNOM can be applied to the microwave regime if certain material constants, like indices of refraction, are revised. Surprisingly, although the resolution of such a microscope is defined by the sharpness of the tip and thus lies in the nanometer range, little research has been done in this field. Keilmann, Knoll, and Kramer ${ }^{195}$ describe the coaxial setup described above ${ }^{172}$ as essentially equivalent to an s-SNOM setup for the microwave regime. Instead of using far-field optics to focus the radiation, they use coaxial cables to direct the fields, which is much more convenient for microwave frequencies. They point out that the resolution achieved is already an order of magnitude higher than the microwave penetration depth of the tip material, indicating that no intrinsic physical mechanism seems to exist that would limit the resolution achievable.

\section{Modulated scattering}

Another technique relies on local scattering of a microwave signal, albeit in a different context. While the scattering techniques treated so far rely on external illumination to probe a passive sample, modulated scattering is intended to probe the near field of an active circuit or antenna. Originally invented in 1955 by several groups ${ }^{196-198}$ to measure the electric near fields around horn antennas, it was recently expanded and theoretically treated by Bokhari et al. ${ }^{199}$ to map tangential near fields over microstrip antennas at about 1.5 GHz. Budka et al. ${ }^{200-203}$ have applied this concept to map 


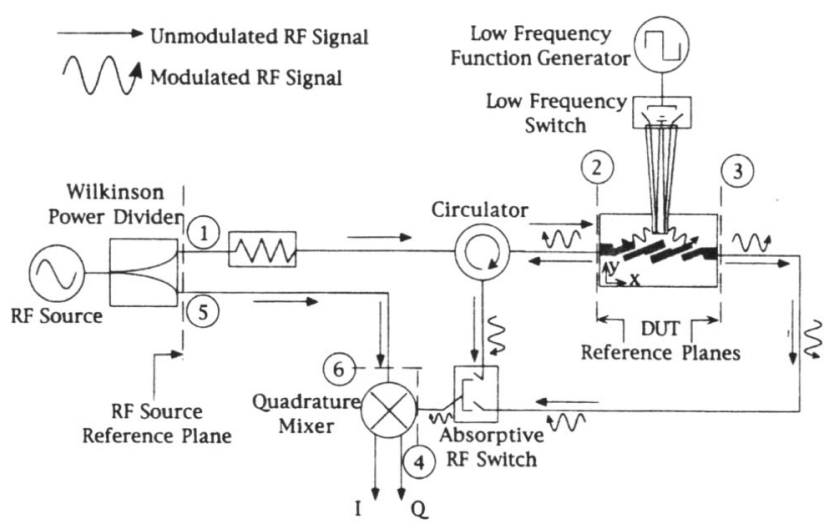

FIG. 12. Microwave circuit electric field imaging setup using modulated scattering (see Ref. 201). This setup allows for detection of reflected or transmitted scattered fields.

the electric near fields of active microwave monolithic integrated circuits (MMICs). In this technique, a miniature monopole or dipole, switched by the slowly modulated bias of a diode mounted between the dipole arms, is used to periodically and locally scatter the microwave signal on a device under test (DUT). Phase and amplitude of this modulated signal are directly proportional to the electric field intercepted at the position of the dipole probe. ${ }^{196}$ Figure 12 shows how the signal can be detected at the input (reflection) or output (transmission) port of the device and differentiated from the bulk-DUT signal by its modulation. Modulated scattering is easy to implement since the probe does not need to carry high-frequency signals, although interpretation of the scattered signal is not always straightforward. Because the modulated signal needs to travel from the point of its origin to one of the ports, it might encounter attenuation due to the features of the DUT that it needs to pass. It was shown ${ }^{200,203}$ that a signal scattered from the far end of a bandpass filter back to the input port appears much less intense if its frequency lies in the rejection band. To circumvent this problem, Budka, Waclawik, and Rebeiz ${ }^{202}$ have proposed a transmission setup that measures the scattered signal from the output port of the device, useful for nonreciprocal DUTs, and a calibration technique has been proposed to de-embed the nonreciprocal element. ${ }^{201}$ Another type of calibration was developed to relate amplitudes of dipole and monopole probes to create a complete vectorial electric field map. ${ }^{201}$ The resolution of this technique is limited by the size of the monopole or dipole and the distance from the sample. Budka and co-workers report using $100 \mu \mathrm{m}$ sized probes, scanned less than $100 \mu \mathrm{m}$ above the circuit without distance feedback, resulting in a resolution of about $100 \mu \mathrm{m}$ over a range of $0.5-18 \mathrm{GHz}$.

While the two scattering techniques presented here are distinctly different, a useful hybrid system could be derived by merging certain advantages of each. Depending on their frequency ranges, MMICs have some important features in the millimeter range. Consequently, modulated scattering does not need to achieve micrometer resolution, although it could benefit from resolutions high enough to image inputs and outputs of the field effect transistors used. In the visible regime, integrated circuits do not abound, and optical tech-

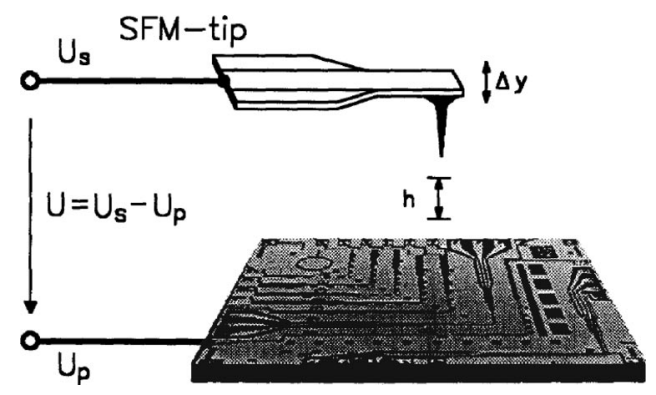

FIG. 13. Principle of high-frequency EFM scanning. The potential difference between DUT and probe produces an electric force that bends the cantilever and can be detected via standard SFM detection means, for example, the deflection of a laser beam off the cantilever (see Ref. 217).

nology allows for good focusing and light detection in free space. Thus, s-SNOM techniques rely on external far-field illumination and are primarily used to scan passive samples. With the advent of photonic band gap (PBG) technology, there is greater emphasis on integrating wavelength multiplexing and other complex tasks into monolithic circuits. Here, an optical analogy to the modulated scattering technique might be a useful diagnostic tool. We envision a system that uses a sharp, conducting s-SNOM tip as scattering probe. Modulation would be achieved by tapping-mode force microscopy, rather than by external switching. This system would provide topography and a local scattering signal while all optical instrumentation could be located at the input or output ports of the PBG structure. While systems using visible light would benefit from out-of-plane far-field detection as in today's s-SNOM, the proposed system seems applicable for $\mathrm{THz}$ and FIR circuits where far-field quasioptical light guiding components are not yet well developed.

\section{High-frequency electrostatic force microscopy}

High-frequency electrostatic force microscopy (HFEFM) is different from the above mechanisms in that a highfrequency electrical signal is converted to a low-frequency mechanical signal that can easily be read out. In practice, a wave form coherent with that on the substrate is injected on a scanning tip, and frequency mixing in the square-law dependence of cantilever force on tip-sample voltage is used to down-convert the wave form to mechanically detectable frequencies. The instrument works by measuring the electrostatic force between the conductive tip of the probe and the sample, typically a circuit, as shown in Fig. 13. A periodic wave form $V_{\text {samp }}$ is applied to the sample, another voltage $V_{\text {prob }}$ to the probe. Both are referenced to a common ground so that the voltage across the gap is $V_{\text {prob }}-V_{\text {samp }}$. The measured electrostatic force is then given ${ }^{204}$ by

$$
\begin{aligned}
F & =\frac{\epsilon_{0} A}{2 z^{2}}\left(V_{\text {prob }}-V_{\text {samp }}\right)^{2} \\
& =\frac{\epsilon_{0} A}{2 z^{2}}\left(V_{\text {prob }}^{2}-2 V_{\text {prob }} V_{\text {samp }}+V_{\text {prob }}^{2}\right),
\end{aligned}
$$

where $F$ is the electrostatic force, $\epsilon_{0}$ is the permittivity of vacuum, $A$ is the effective tip area and $z$ is the effective tip-sample separation. The cross product generates mixing 
terms that include the difference frequency, which can be chosen to be sufficiently low to fall below the fundamental mechanical resonance of the cantilever. The frequencies can also be chosen such that higher harmonics as well as the sum frequency are too high for mechanical detection. Two different detection approaches have been proposed. The broadband heterodyne technique uses a sampling wave form that is repetitively applied to the probe with a slightly different frequency from that of the sample. A pulse signal is used as the sampling wave form, resulting in a spectrum of force components that should all fall within the cantilever's mechanical bandwidth. The second technique is narrow band detection in which a sine wave signal is applied to the probe, resulting in only one force component.

A few groups ${ }^{204-220}$ have worked on this technique. It was invented in 1992 by Hou, Ho, and Bloom, ${ }^{205}$ who accomplished frequency mixing up to $20 \mathrm{GHz}$. Later, wave form measurements were performed on a $\mathrm{MHz}$ complementary metal-oxide-semiconductor (CMOS) chip, ${ }^{206,207}$ followed by heterodyne scanning at $3.2 \mathrm{GHz}$ on a $\mathrm{CPW}$ circuit. $^{204}$ Bridges et al. ${ }^{209-212}$ started with proof-of-principle experiments for heterodyne detection in the $\mathrm{kHz}$ range, ${ }^{209}$ demonstrated Mbit digital pattern extraction ${ }^{210}$ and later presented vector-voltage circuit probing at $10 \mathrm{GHz} .^{211}$ Later a nulling method was developed ${ }^{209}$ that allowed for direct measurements over passivated structures. Recently, a pulse width modulation technique was developed, ${ }^{212}$ which enhances measurement resolution, enabling $2 \mathrm{D}$ voltage maps to be presented. Böhm et al. ${ }^{213-220}$ presented a system in $1994^{214}$ which was able to resolve signals up to $15 \mathrm{GHz}$. Leyk et al. $^{215}$ presented measurements up to $104 \mathrm{GHz}$, aided by NLTLs. ${ }^{221}$ Probes with integrated CPWs have been presented $^{218}$ as well as 2D voltage maps at $1 \mathrm{GHz}^{219}$ In 1996, the researchers presented two-dimensional scans of MMICs, ${ }^{220}$ measuring both voltage and phase at up to 10 $\mathrm{GHz}$ with $160 \mathrm{~nm}$ scan steps. Since the tip responds to van der Waals interactions when in nanometer range to the surface, a first scan in SFM mode reveals topography. To acquire voltage maps, the tip is lifted to a fixed height, typically $50 \mathrm{~nm}$, above the device where long-range interactions like the Coulomb force become dominant. ${ }^{216,219,220}$ Hong et $a l .{ }^{208}$ have developed a feedback technique that allows for simultaneous topography and voltage acquisition. They applied a probing voltage consisting of a train of voltage pulses, modulated by a sine wave with frequency $f_{0}$. They found it possible to extract the sample voltage signal through the signal component at $f_{0}$, while the component at $2 f_{0}$ contained the topographic information of the sample via $\partial C / \partial z$.

There are a few techniques that are related to HFEFM, particularly scanning kelvin probe force microscopy (KFM). ${ }^{222}$ Here, an ac signal with frequency $f$ is applied to the cantilever and tip while the sample holder is kept at ground potential. The resulting electrostatic force on the cantilever has nonlinear force-voltage dependence and thus generates components with $f$ and $2 f$. Commonly, the component with frequency $2 f$ is used in a feedback loop to keep tipsample capacitance constant. The other force component can then be used to map the contact potential between tip and

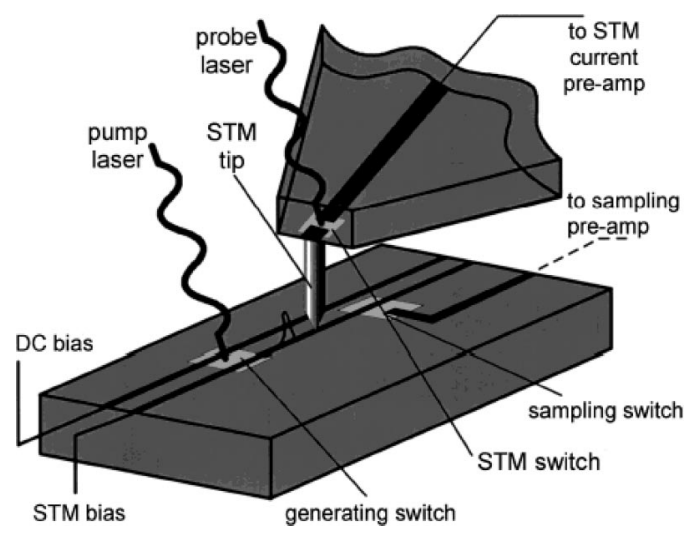

FIG. 14. Localized pump and probe setup that uses photoconductive switches to trigger a pulse on the DUT and to probe the instantaneous voltage on the DUT through the tip (see Ref. 224).

sample. KFM is much used in chemical physics but it is not inherently a high-frequency technique. Therefore, it will not be discussed in any further detail.

\section{Pump and probe experiments}

Two different flavors of ultrafast scanning tunneling microscopy were invented independently by Weiss et al. ${ }^{223,224}$ and Nunes and Freeman ${ }^{225}$ in 1993 . Both techniques rely on pump and probe experiments for highest temporal resolution. The pump pulse is used to start the dynamic process or sample response of interest while the probe pulse is used to interrogate the instantaneous sample state. By delaying the arrival time of the probe pulse with respect to the pump pulse, the fast time response of the system can be mapped out with a resolution roughly equal to the width of each pulse. Switching of voltages with picosecond resolution is commonly done by using femtosecond lasers that repetitively illuminate photoconductive (PC) switches that enable a short electrical pulse to propagate. While this ensures high temporal resolution, using a sharp tip gives the additional benefit of high spatial resolution. The nonlinear current versus voltage tunneling characteristics are employed to measure the picosecond cross correlation. In the setup of Nunes, pump and probe travel along one transmission line while Weiss employs the more common setup of pumping the sample and probing the tunneling tip as shown in Fig. 14. Takeuchi, Kasahara, and Mizuhara ${ }^{226-228}$ proposed a STM system that uses a PC switch on the probe, measuring a free-running signal on a circuit. The light source was a pulsed laser diode, synchronized to the circuit signal. A Pt/Ir probe tip was used, resulting in temporal resolutions down to $160 \mathrm{ps}$. By changing to a pump/probe setup, using a femtosecond laser and employing a low-temperature GaAs (LT-GaAs) probe with integrated metal leads and PC switch, temporal resolution could be improved to $2 \mathrm{ps}$.

A related pump-probe technique was introduced by Kim, Williamson, and Nees et al. ${ }^{229}$ that relies on direct conduction through a tip. They brought a probe with Ti tip, PC switch and electric lead into contact with a transmission line. The convolution between pump pulse and probe pulse could be mapped out by probing the instantaneous electric field at the tip position by opening the PC switch and collecting the 


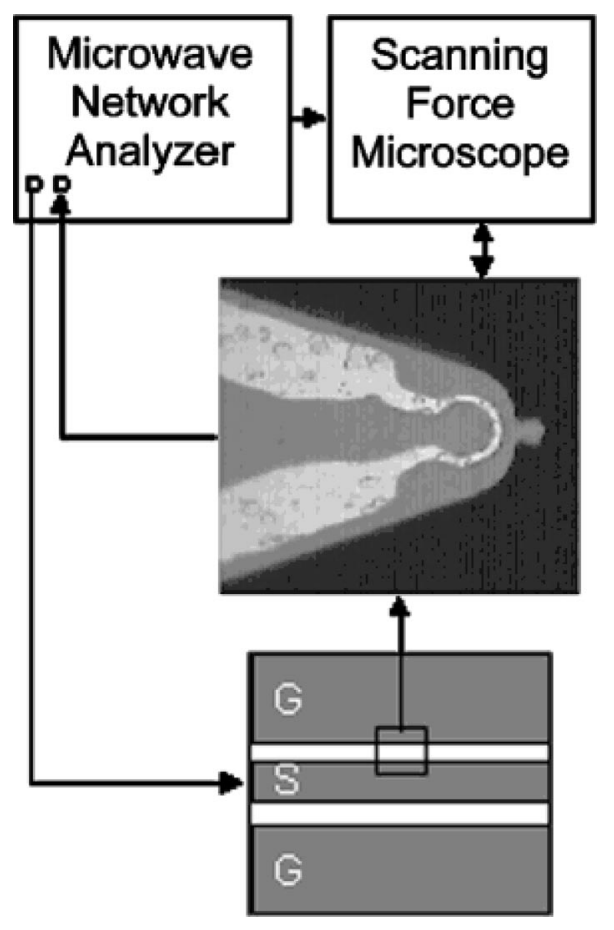

FIG. 15. Experimental setup of a microscopic magnetic field probe. Shown is the cantilever with sensing loop and scanning tip on the right, DUT below and the instrumentation above (see Ref. 239).

signal transmitted through the tip. The same group improved on this concept by integrating an LT-GaAs tip and a PC switch with a SFM setup. ${ }^{230}$ Takeuchi and Mizuhara ${ }^{231}$ presented a similar setup, employing SFM feedback, LT-GaAs tip and pump/probe data acquisition. A propagating pulse on a metal strip was mapped in $2 \mathrm{D}$ and observed in sequential images with 0.8 ps time intervals. Oesterschulze et al. ${ }^{232,233}$ have also reported on a SFM setup with Lt-GaAs probe and PC switches. Here, contact and noncontact images of a voltage pulse on a coplanar stripline were presented with picosecond temporal resolution.

Pfeifer et $a l .{ }^{234,235}$ used freely positionable PC sampling probes that could act as detectors and as generators for ultrafast electric signals. While these experiments used a typical pump/probe setup, Löffler et al. ${ }^{236}$ also presented a system that can measure voltage wave forms of a free-running microwave circuit at up to $75 \mathrm{GHz}$ by changing the repetition rate of the probe pulse to track the free-running microwave DUT. This technique is also applicable to both direct and indirect electro-optic sampling, ${ }^{237}$ using light beams as well as probes.

\section{Magnetic field probes and microwave SQUIDS}

Although the first experimental near-field microscopes were magnetic microscopes, ${ }^{9,10}$ all microscopic microwave probes we have discussed so far measure electric field components. There are, however, numerous applications for highfrequency magnetic measurements, ranging from examining superconducting thin films to locating shorts on integrated circuits. While magnetic force microscopes are designed to measure static fields, ${ }^{238}$ few instruments allow for microwave magnetic field imaging. One suitable instrument pre- sented by our group ${ }^{239}$ consists of a $6 \mu \mathrm{m}$ loop antenna, defined on a SFM cantilever, capable of probing components primarily normal to the sample plane as seen in Fig. 15. This instrument, which can operate at environmental conditions, measured the magnetic field across a CPW at $10 \mathrm{GHz}$. Since this probe is only an antenna, device performance is primarily limited by the external instrumentation. In the measurement setup used, the device noise at $10 \mathrm{GHz}$ was comparable to that of a superconducting quantum interference device (SQUID) at dc. Another technique recently published by Lee et al. ${ }^{240}$ uses a closed loop tip that connects the inner and outer conductors of a driven resonant open coaxial guide. The experiments, carried out at $6 \mathrm{GHz}$ and with a resolution of $200 \mu \mathrm{m}$, were performed by keeping track of one of the resonant frequencies of the coaxial resonator. Perturbations from the sample shift the resonance frequency; this frequency shift was monitored and imaged. It was possible to observe contrast between ferromagnetic and paramagnetic materials using this probe. Recent theoretical investigation ${ }^{241}$ have shown that it might be possible to use a s-SNOM setup to measure magnetic properties of a sample via the Kerr effect. The final technique for magnetic measurements presented here is based on SQUIDs, which have an inherent advantage in sensitivity over the loop probes because their minimum-detectable field does not scale with frequency. Furthermore, SQUIDs provide quantitative data in contrast to most other scanned probe technologies. Their inherent disadvantage is that no superconducting materials are available at ambient temperatures, restricting their application to samples that can be cryogenically cooled, or to imaging that can take place near a cold finger. The resolution of SQUID microscopes is currently limited to about $5 \mu \mathrm{m}$ by the size of the inner hole. ${ }^{242,243}$ While SQUIDs have been primarily used for dc magnetic measurements, it is also possible to apply them for ac. Black et al. ${ }^{244}$ have shown that it is possible to perform eddy current microscopy with these probes when applying a driving current at $26-100 \mathrm{kHz}$. This technique, reaching a resolution of $80 \mu \mathrm{m}$, is useful to detect small regions of nonmagnetic conducting materials. SQUIDs can also be used for microwave frequencies as shown by Black et al. ${ }^{245-247}$ Detection of microwave signals up to 125 $\mathrm{GHz}^{247}$ at $77 \mathrm{~K}$ is accomplished by using the nonlinearity of the voltage-flux characteristics of the SQUID to rectify the millimeter wave fields. The setup does not use a feedback system and the spatial resolution is about $30 \mu \mathrm{m}$.

\section{B. Resonant probes}

In some setups already discussed, cavities have been used to shield the scanning tip and sample from their surroundings. ${ }^{179}$ In this case, the resonant behavior of a closed cavity was undesirable. In many microwave applications, however, resonant structures are essential because they allow localization of high field areas. They are very efficient in the frequency band for which they were designed, since the signal-to-noise ratio in a resonator structure increases with resonator quality factor $Q$. This increase in sensitivity and field strength is accompanied by a narrower frequency band, with the drop in amplitude depending on $Q$, which 
results from the shift in resonant frequency with different dielectric environments. Consequently, resonance frequency and amplitude tracking are employed. Thus, resonant structures are often more sensitive and efficient in transmission but less broadband than the techniques discussed so far. Most of the instruments presented here do not have feedback systems and do not achieve subwavelength resolution unless otherwise noted. Amplitude modulation of the microwave signal and consequent lock-in detection of the rectified detected signal is commonly employed to increase signal-tonoise ratio.

\section{Resonant slots}

Golosovsky and Davidov ${ }^{248}$ introduced a novel resistivity microscope that uses a narrow resonant slot in a rectangular hollow waveguide as near-field source. Building on the original work by Ash and Nicholls, ${ }^{12}$ they found a geometry that allows for much higher transmission than using a circular aperture. It is well known from basic electromagnetic theory that a sheet of metal separating two parts of a rectangular waveguide can be nearly transparent for incident fundamental transverse electric (TE) waves if it has a slot cut into its center with the right proportions. By moving this sheet with slot to the end of the waveguide, Golosovsky and Davidov produced a near-field source with high transmission coefficient and a resolution limited by the slot dimensions. The slot resonator has a relatively low $Q$, which results in acceptable bandwidth, operated from 10 to $80 \mathrm{GHz}$. Because of the slot shape, this probe has unequal resolution in $x$ and $y$ unlike all other techniques presented so far. The authors propose to use deconvolution techniques, similar to twodimension projection reconstruction used in magnetic resonance imaging, to compute a $2 \mathrm{D}$ resistivity map with resolution better than $100 \mu \mathrm{m}$ in $x$ and $y$. Another image reconstruction technique developed for near-field microwave microscopy involves a blind deconvolution approach ${ }^{249}$ because it is challenging to determine the point spread function of the probe from physical data.

Golosovsky further developed the instrument ${ }^{250}$ for higher spatial resolution. The apex of the probe was replaced by a dielectric piece, confining the microwaves. Then this probe tip was given a curved surface where the slot was positioned perpendicular to the axis of curvature. This way, the outer parts of the slot were spaced farther from the sample, contributing less to the signal, resulting in 10-100 $\mu \mathrm{m}$ resolution.

Lann, Golosovsky, and Davidov ${ }^{251}$ modified the system further to incorporate a cylindrical waveguide at the probing end with two perpendicular slits at the convex, dielectric apex of the probe as seen in Fig. 16. This instrument was used for near-field polarimetry: microwaves between 82 and $96 \mathrm{GHz}$ were directed to the probe, polarized along one slot direction. The reflected signal, which contained both polarization directions, originating from the crossed slits, was separated and independently detected.

Another improvement to the original instrument was made by incorporating distance feedback. ${ }^{252}$ It was found that it is possible to use high frequency at $82 \mathrm{GHz}$ for image acquisition while a simultaneous low-frequency signal at 5

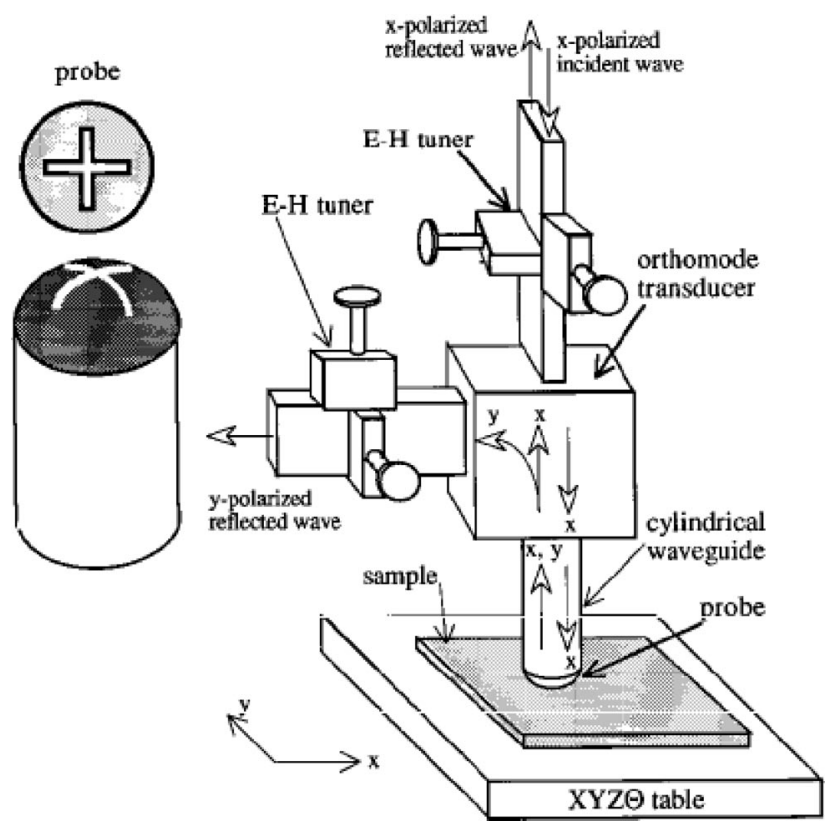

FIG. 16. Design of the resonant cross-slit probe employed by Lann, Bolososky, and Davidov (see Ref. 251).

$\mathrm{MHz}$ was used for capacitance distance control. This was possible because the electric field lines behave very differently for the two frequencies. The lines stretch from one side of the slit to the other at high frequencies, thus they are almost tangential at the surface and induce currents in the sample. This inductive behavior is very different from the capacitive mode at low frequencies. Because the probe is much smaller than the low-frequency wavelength, the surface of the probe is equipotential and field lines stretch from the probe normally onto the sample. It was found that the probe-sample capacitance at $5 \mathrm{MHz}$ is almost insensitive to the sample resistance, thus it could be used for distance control.

Abu-Teir et al. ${ }^{253}$ lithographically defined the slit on the convex probe head, using thin threads as masks for metal evaporation. The instrument, operated at $25-30 \mathrm{GHz}$, had a resolution of $1-10 \mu \mathrm{m}$ and a low impedance of $20 \Omega$. While this system does not have distance feedback, strong dependence of the reflected signal on probe-sample distance was observed. This is to be expected as the resonance frequency and amplitude shift (often strongly) in the presence of a sample.

This strong dependence was also found in a similar probe design employed by Nozokido et al. ${ }^{254,255}$ Here, the rectangular waveguide is tapered down to the size of the slot, resulting in a calculated 20\% transmission efficiency. Experiments at $60 \mathrm{GHz}$ show that image resolution equal to the slit width $(80 \mu \mathrm{m})$ is achieved if the sample is held within $2 \mu \mathrm{m}$ of the sample. Because of the relatively wide bandwidth design of the probe, it was possible to investigate transition phenomena of photoexcited free carriers in $\mathrm{Si}$ with $0.4 \mathrm{~ns}$ response time. A special scanning scheme was employed to facilitate deconvolution of probe shape and real sample response. 


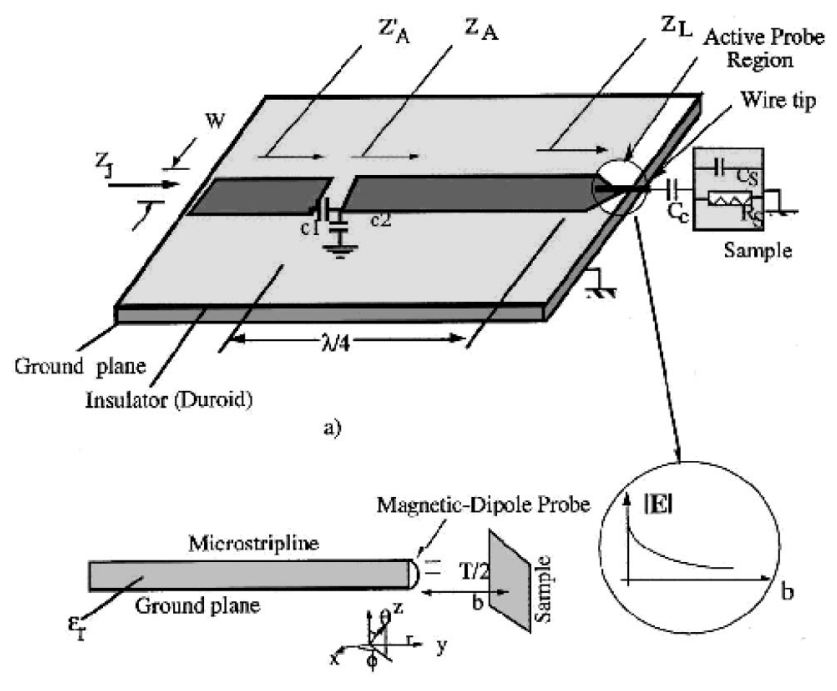

b)

FIG. 17. (a) Design of the microstrip line resonator and probe employed by Tabib-Azar et al. (see Ref. 258) for electric field measurements. The inset shows that evanescent waves extend from the tapered tip. (b) Magnetic field probe configuration using a short length of current carrying wire.

\section{Strip line and microstrip line resonators}

Tabib-Azar, Shoemaker, and Harris have used a strip line or microstrip line resonator for near-field microwave microscopy in a series of publications. The initial experiment ${ }^{256}$ employed a microstrip line (a signal line on top of a substrate, backed by a ground plane) feeding a $\lambda / 4$ microstrip line resonator. This is achieved by disconnecting and capacitively coupling the strip line from a $\lambda / 4$ piece of microstrip. The signal conductor of the resonator was tapered to a fine point at the substrate end and connected to a short wire that constitutes the probe. The probe wire was either connected to the back short (magnetic probe) or an open circuit (electric probe), as shown in Fig. 17. Resolution of this noncontact probe was $100-150 \mu \mathrm{m}$.

A slightly different geometry ${ }^{257}$ that uses a tapered strip line resonator (signal line sandwiched between two substrates and backed by two ground planes) as probe was also introduced. The resonator is designed as previously, by capacitively coupling to the stripline, and a sharpened stainless steel wire was used as a probe tip. Because the strong distance dependence of the reflected signal is also present in this resonant setup, an independent distance control was employed. The researchers used a reflectance-compensated fiberoptic distance sensor next to the tip and maintained the probe-sample separation with an accuracy of $0.4 \mu \mathrm{m}$. A synchronous detection scheme was employed in which the sample was vibrated at $100 \mathrm{~Hz}$ and the modulated signal detected using a lock-in amplifier. The researchers claim 0.4 $\mu \mathrm{m}$ spatial resolution at $1 \mathrm{GHz}$ for this instrument. It was tested on a variety of samples ${ }^{258,259}$ and probes of this design for $10 \mathrm{GHz}$ were microfabricated on high-resistivity silicon and trimmed for a high external quality factor of over $13000 .^{260}$ The bandwidth of these probes was reported to be $5 \mathrm{MHz},{ }^{259}$ setting a lower limit of $0.2 \mu \mathrm{s}$ on the probe's response time. Some deconvolution, based on the field pro-

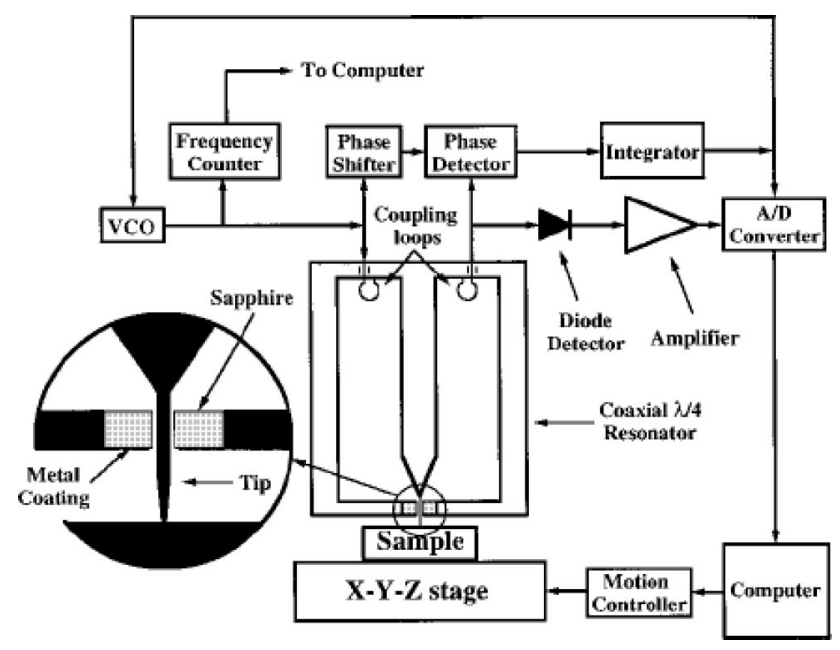

FIG. 18. Resonant SNMM setup using a coaxial cavity. Coupling loops are used to close energy in and out of the resonator. The resolution of this instrument is determined by the sharpness of the tip and the opening size for the extending tip in the resonator wall (see Ref. 263).

file of a line scan over a wire, was employed in one publication. ${ }^{258}$

\section{Coaxial cavity resonator}

Designs of microwave near-field probes incorporating coaxial resonators have been published by Cho, Kirihara, and Saeki ${ }^{261}$ and Wei et al. ${ }^{262}$ in 1996. Cho's design, used for imaging of ferroelectric materials, consists of an open coax resonator that contains the specimen between the resonator and a backing electrode. This design is limited in resolution to the millimeter range, using frequencies around $1 \mathrm{GHz}$. In the design by Wei et al. ${ }^{262}$ a STM-like tip rather than an aperture is used as a point-like field emitter. The sharp tip results from tapering the center conductor of the $\lambda / 4$ coaxial cavity. The cavity is closed all around except for a $1 \mathrm{~mm}$ sized hole, concentric to the probe tip, which extends beyond the shielding. This enabled the instrument to reach a resolution of $5 \mu \mathrm{m}$ when in contact mode, pushed into physical contact by gravitational force. Later, lower contact force was achieved by pressing the sample against the probe via a soft cantilever. Thus, to image conducting samples, the samples had to be coated with a thin insulating layer. The operating frequency was between a few hundred $\mathrm{MHz}$ and $10 \mathrm{GHz}$. The instrument was improved by Gao et al. ${ }^{263}$ to achieve a spatial resolution of $100 \mathrm{~nm}$ by reducing the size of the aperture and sharpening the probing tip. Figure 18 shows that a ring of sapphire was inserted into the hole, with an inner diameter of 100-200 $\mu \mathrm{m}$, decreasing the aperture size. The outer side of this ring was coated with $1 \mu \mathrm{m}$ of metal, to shield far-field propagating components. This setup was chosen because the thin metal coating, which is as thin as the skin depth, reduced losses resulting from the proximity of the center wire and the shielding wall, keeping the $Q$ factor as high as possible. It was used to image dielectric-constant profiles and ferroelectric domains. ${ }^{264}$ The researchers have succeeded in quantitatively mapping the third order dielectric constant in a ferroelectric domain structure. ${ }^{265}$ The instrument was also implemented in a low-temperature cryostat ${ }^{266}$ 
where it was used to image superconducting $\mathrm{YBa}_{2} \mathrm{Cu}_{3} \mathrm{O}_{7-x}$ (YBCO) films. Further experimental work, accompanied by theoretical model analysis, has produced quantitative microscopy of dielectric and conducting properties of passive samples. ${ }^{267,268}$ As part of their theoretical modeling, the researchers have found that the intrinsic spatial resolution of their microwave near-field microscope, an important figure of merit, is dependent on the dielectric constant of the sample. They propose that as a figure of merit, the ratio of wavelength inside the sample to spatial resolution is more informative. ${ }^{267}$ Since the wavelength of microwaves in metals is several orders of magnitude smaller than in air, metals are not suitable for the determination of resolution. It is interesting to observe that many groups have used evaporated metal targets to determine the spatial resolution of their design. Thus, when comparing different techniques for microwave microscopy of passive samples, attention should also be paid to the material used for testing the resolution.

In order to decrease tip damage due to excessive forces on the tip, and in order to be able to image unprepared conducting samples, Duewer et al. ${ }^{269}$ introduced a tip-sample feedback scheme based on regulating the resonance frequency of the cavity to maintain a constant separation. For small distances, where the tip-sample separation $g$ is smaller than the tip radius $R_{0}$, the frequency shift was found to be

$$
\frac{\Delta f_{r}}{f_{r}}=-1.14 A \log \left(\frac{g}{R_{0}}\right),
$$

where $A$ is a geometric factor and $f_{r}$ is the resonance frequency.

In this experiment, conductivity was measured simultaneously via the amplitude of the cavity resonance.

\section{Open resonant coaxial resonator}

Wellstood and Anlage have presented a range of publications focused on the application of another kind of microwave near-field probe. In its first realization by Vlahacos et al.,${ }^{270}$ this open rigid coaxial probe with an inner diameter of $100 \mu \mathrm{m}$ was used in reflection or collection mode in the frequency range of 7.5-12.4 GHz. It closely resembles the coaxial open-ended near-field probes discussed at the beginning of this chapter, except that the coaxial piece was further miniaturized in diameter. The rigid coaxial probe can be viewed as a resonant coaxial transmission line terminated at the sample end with a capacitor. This is the capacitance between the inner coaxial lead and the sample and thus is responsible for microwave contrast. The diameter of the inner conductor of the probe was subsequently reduced to 12 $\mu \mathrm{m} .{ }^{271}$ With these probes, resonances occurred about every $125 \mathrm{MHz}$, allowing for a near-continuous choice of frequency. To circumvent complications from the convolution of frequency shift and the simultaneous change in $Q$, a frequency-following circuit was developed by Steinhauer et al. ${ }^{272}$ The entire setup for this instrument can be seen in Fig. 19. It was applied to image surface resistance of metallic samples. Vlahacos et l. $^{273}$ used the same principle and related the frequency shift quantitatively to the probe-sample separation. Thus, it was possible to measure topography of

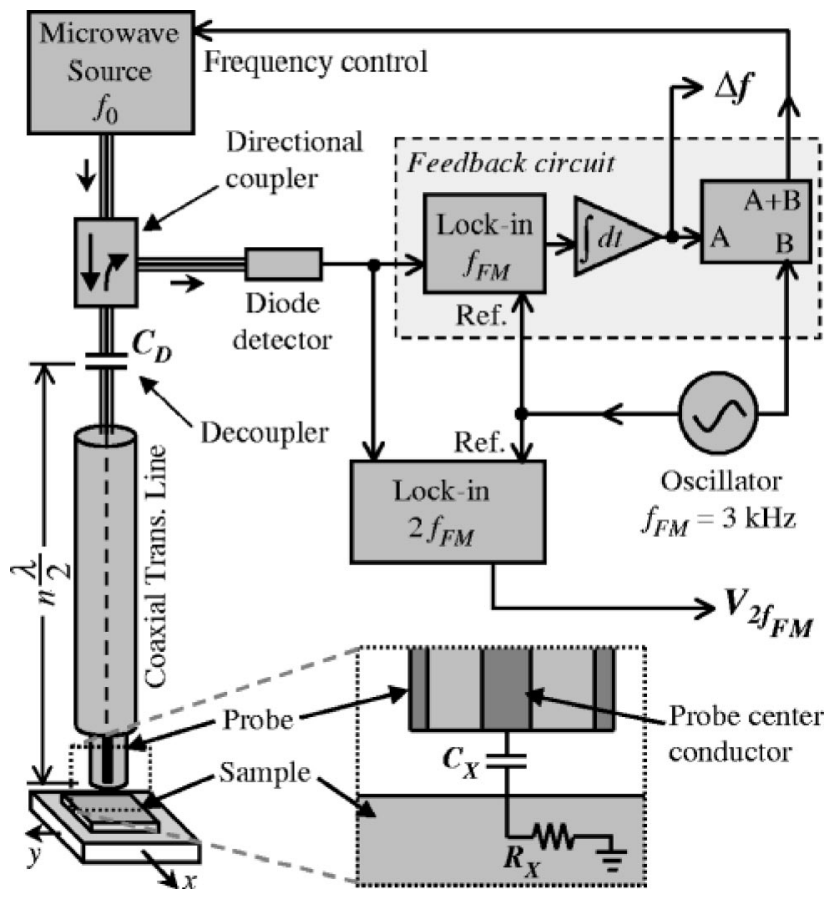

FIG. 19. Schematic of the SNMM employed by Steinhauer et al. (see Ref. 289). The probe consists of a resonant open coaxial line. The inset shows the interaction between probe and sample, represented by a capacitance $C_{x}$ and a resistance $R_{x}$.

an evenly conducting sample without employing distance feedback techniques. Anlage et al. ${ }^{274}$ used a sharp STM tip as an extension of the coaxial center conductor to increase spatial resolution and achieved resolution down to $1 \mu \mathrm{m}$ when scanning in contact. A very similar setup was then used by Steinhauer et al. ${ }^{275}$ and Vlahacos et al. ${ }^{276}$ to quantitatively image microwave permittivity and tunability in thin films. The same group ${ }^{277}$ used a coaxial probe as a passive sensor for imaging microwave electric fields on an open transmission line. Here, the resonator consisted of a piece of open coaxial cable, connected to the probe via a directional coupler. Dutta et al. ${ }^{277}$ showed that it is possible to measure different components of the electric field of the standing wave pattern by orienting the coaxial probe differently with

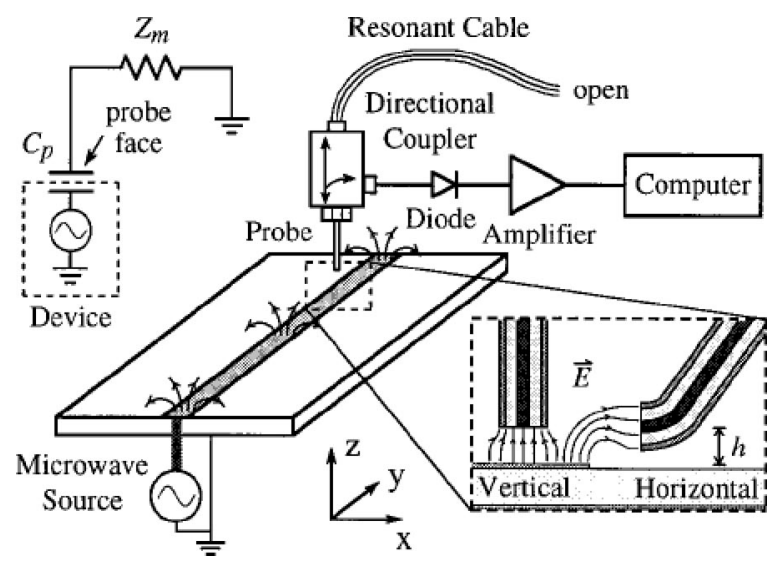

FIG. 20. The passive resonant coax probe employed by Dutta et al. (see Ref. 277). The inset on the upper left shows a model of the interaction of the device with the probe. The inset in the lower right shows how the probe orientation can be changed to measure different field components. 
respect to the DUT and using it as a passive probe as shown in Fig. 20. The measurements at $8 \mathrm{GHz}$ were carried out with a spatial resolution of about $200 \mu \mathrm{m}$.

\section{APPLICATIONS OF MICROWAVE NEAR-FIELD MICROSCOPY}

While there are many different techniques for microwave near-field microscopy, there are equally many applications. Some of these, like nondestructive testing (NDT) of passive material, have been applied for decades while others, e.g., characterization of active integrated circuits, are recent developments. Still others like the sensing and monitoring of biological samples are not yet well developed.

Imaging of passive and active samples is different in that passive samples interact with the local probe through their conductivity, thus a probing field is always required. For active samples, the near-field probe can act as an entirely passive collector or scatterer of electromagnetic radiation. In the case of pump-probe and sampling experiments, the probe uses the interaction between an injected signal and the sample signal to produce an image. A summary of the applications described in the articles that we have listed so far follows.

\section{A. Passive samples}

Microwave and millimeter wave NDT has many applications in evaluative material probing (for example, measuring thickness) and quality control. ${ }^{278}$ Polarization properties of microwave signals can be used to increase measurement sensitivity to defects in a certain direction. Microwave NDT is (by definition) minimally invasive and can be conducted in air, liquid or under vacuum conditions. It can be used for superconducting, conducting, semiconducting and insulating samples. Since microwave near-field microscopes measure conductivity changes, they can be applied for moisture content measurements. Other applications include disbond, void and delamination detection in stratified media. Stress and fatigue surface cracks in metals can be detected underneath various coatings including paint. ${ }^{151}$

\section{Ferroelectric domains}

Ferroelectric domains are of interest as dielectric layers of capacitors in microelectronics due to their large dielectric constant. They have also found applications in quasiphasematched nonlinear optics. Thus, there is considerable interest in imaging microscopic ferroelectric domains using a nondestructive technique. Ferroelectric domains can be imaged using microwave fields by taking advantage of the nonlinear components in the dielectric constant for these materials. Some researchers ${ }^{261}$ base their contrast mechanism on alternating capacitance variations related to the third-order dielectric constant. Others ${ }^{265}$ introduced a low-frequency oscillating voltage between sample and substrate that modulates the effective dielectric constant of the sample. The slight shift of the resonance frequency in the coaxial resonator probe could be related to this variation in dielectric constant, allowing for quantitative mapping of the third order dielectric constant in a ferroelectric domain structure as seen in Fig. 21. Another publication by the group of Xiang ${ }^{262}$

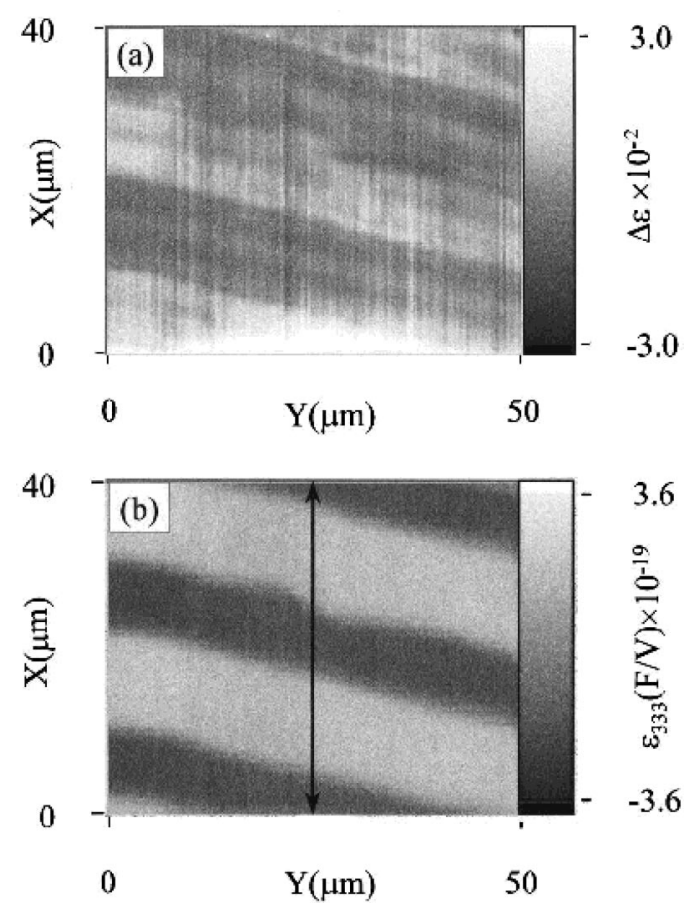

FIG. 21. Linear dielectric constant image (a) and nonlinear third order dielectric constant image (b) of the same area of a $\mathrm{LiNbO}_{3}$ single crystal with periodically polarized ferroelectric domains, recorded using a coaxial resonator SNMM (see Ref. 263).

showed simultaneous recording of dielectric constant profiles and ferroelectric domains. The image of the resonant frequency reflects variations in the dielectric constant associated with changes in the dopant level. The periodic variation in the dopant level induces the formation of ferroelectric domains with alternating polarization. Thus, variations in the resonance frequency of the resonant probe coincide with the location of the domains. At the same time, the $Q$ factor of the resonator was recorded. Its image corresponds to losses in microwave energy, which occur largely at the ferroelectric domain boundaries. By correlating the two sets of data, it was possible to observe a defect in the periodic domain structure.

\section{Resistivity, dielectric constants, and chemical contrast}

The principle for resistivity microscopy is based on the fact that the reflection of electromagnetic waves from conducting surfaces is determined by their resistivity. Therefore, measuring the reflected amplitude and phase of a near-field probe yields a resistivity map. However, the reflection coefficient is also strongly influenced by the distance of probe to surface, thus a constant probe-sample gap is important. For thick conducting layers, much thicker than the skin depth, surface impedance is measured while thin layers yield sheet resistance.

Microwaves have certain advantages ${ }^{258}$ over ultrasonic testing of insulators. Because they have relatively high penetration depth, they can image bulk properties. They have good transmission across solid-air boundaries unlike ultrasound. Unlike ultrasound measurements, microwaves can be 

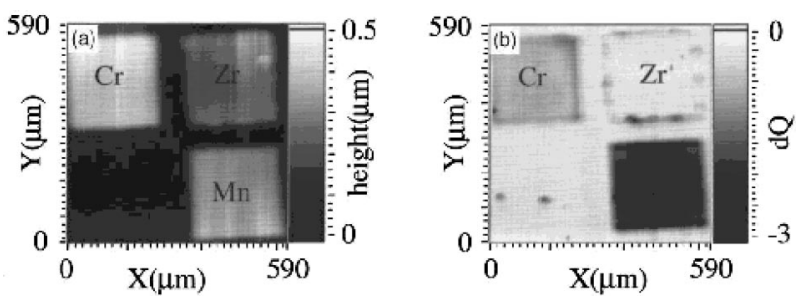

FIG. 22. Topographic (a) and loss (b) images of $\mathrm{Cr}, \mathrm{Mn}$, and $\mathrm{Zr}$ squares on thin Pt film on a Si substrate, recorded using the coaxial resonator SNMM developed by Duewer et al. (see Ref. 269). Lighter regions correspond to elevated regions in the topographic image while they correspond to higher conductivities in the loss image.

applied to high temperature testing of most materials. In poorly conducting materials, they are useful to image subsurface features.

Thus, there is potential in nondestructive testing of airplane parts, imaging of defects and nonuniformities in composite materials and imaging of impurities and residual stress in semiconductors. Residual stress in metals and semiconductors causes an increase in random carrier scattering, resulting in lower local conductivities in the stressed regions. ${ }^{258}$ By rigorous modeling of the probe-sample interactions, Gao, Xiang, and Duewer ${ }^{267,268}$ have produced quantitative results on a submicron scale. Topographic and loss images of metal patches, reported by Duewer et al. ${ }^{269}$ can be seen in Fig. 22. Steinhauer et al. ${ }^{275,276}$ also reported quantitative measurements of permittivity of thin films. Golosovsky, Galkin, and Davidov ${ }^{250}$ state that the reflectivity image acquired is directly related to the resistivity map. The reflection coefficient of a plane wave from a conducting surface is

$$
\Gamma=\left(\frac{Z_{s}-Z_{0}}{Z_{s}+Z_{0}}\right)
$$

where $Z_{0}$ is the characteristic impedance of the line and $Z_{s}$ is the surface impedance, which is connected directly to the conductivity

$$
Z_{s}=\sqrt{\frac{i \omega \mu_{0}}{\sigma+i \omega \epsilon}}
$$

where $\omega$ is the radial frequency, $\mu_{0}$ is the permeability of free space and $\epsilon$ is the dielectric constant. Knoll and a)

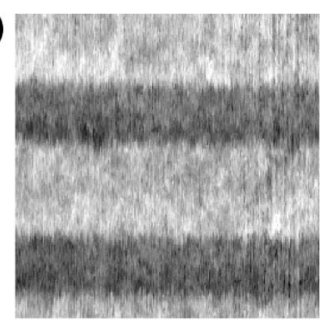

b)

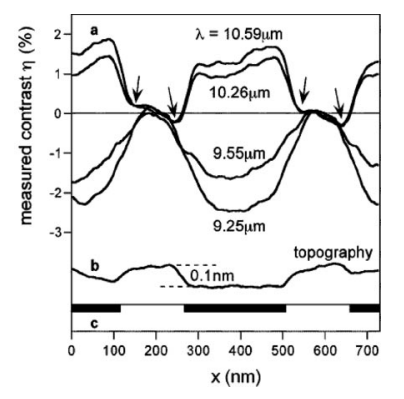

FIG. 23. (a) Infrared s-SNOM image of a flat Si sample with subsurface doping (bright), taken at $\lambda=10.59 \mu \mathrm{m}$. (b) Line scans taken across doping stripes, measured at four different wavelengths, demonstrating $30 \mathrm{~nm}$ spatial resolution and a contrast reversal when the infrared wavelength tunes through the induced plasmon resonance, here at about $\lambda=10 \mu \mathrm{m}$. Topography and designed width of doping stripes are shown below (see Ref. 132).
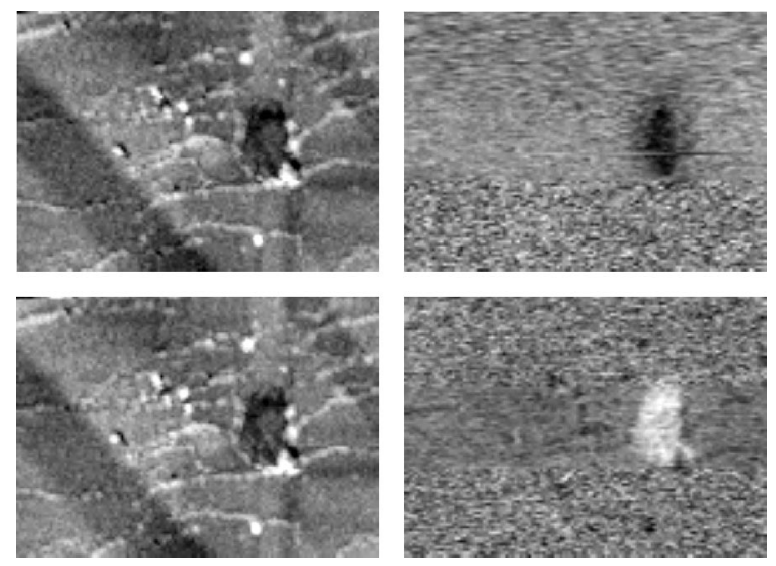

FIG. 24. Simultaneously acquired topography (left) and infrared images (right) of PS embedded in PMMA using an s-SNOM (see Ref. 94). The upper row is recorded with an illumination wavelength of $\lambda=9.68 \mu \mathrm{m}$, where PS has an absorption maximum. The lower row shows the same spot, illuminated at the PMMA absorption maximum wavelength of $\lambda=10.17$ $\mu \mathrm{m}$. The field of view is $3.5 \times 2.5 \mu \mathrm{m}$.

Keilmann ${ }^{132}$ have shown that it is possible to map conductivity of a $\mathrm{Si}$ sample with nanometer resolution using a scattering-type near-field microscope and $10.6 \mu \mathrm{m}$ radiation as shown in Fig. 23. The same researchers ${ }^{94}$ have reported contrast between polystyrene (PS) and polymethylmethacrylate (PMMA) on the nanometer scale using the same instrument and two wavelengths around $10 \mu \mathrm{m}$, (Fig. 24).

\section{High-T $T_{c}$ superconductors}

An important application of evanescent microwave probing is to measure spatially resolved surface impedance of high temperature superconductor films. By finding superconducting and normally conducting features on super conducting thin films, it is possible to localize defects and evaluate film quality. In the case of YBCO films, their electronic properties are highly sensitive to stoichiometric changes, especially small variations in oxygen content. Local offstoichiometric areas can occur at very small scales, emphasizing the need for local measurement methods. The contrast mechanism is based on variations in microwave absorption when keeping the sample close to the critical temperature. ${ }^{266}$ Others employ room temperature normal-state measurements ${ }^{250}$ and relate them to the film's superconducting properties. One method not previously discussed uses a dielectric resonator, made of low-loss single crystal rutile $\left(\mathrm{TiO}_{2}\right)$ and fed via subminiature "A" (SMA) connections to sense sample surface impedance with millimeter resolution. ${ }^{279}$ Anlage et al. ${ }^{271}$ have imaged the fields on an active YBCO thin film microstrip circuit at room and cryogenic temperatures and also showed sheet resistance measurements of YBCO at room temperature. ${ }^{274}$

\section{Chemical and environmental sensing}

Tabib-Azar et al. have used evanescent microwave probes for several nontraditional probing applications. These include transient and steady state thermography on semiconducting materials ${ }^{280}$ with $0.01 \mathrm{~K}$ thermal and $1 \mu$ s temporal resolution. Another application employs physiosorption of 


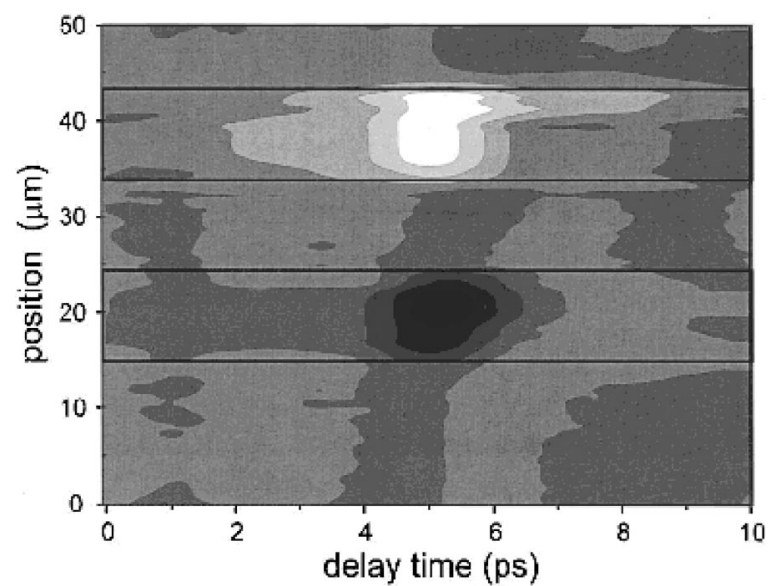

FIG. 25. Spatio-temporal evolution of a voltage pulse between two contacts of a biased coplanar strip line, outlined by black horizontal lines. Vertical line scans were performed, recording the electric field at each point with the same delay time. Changing this delay for each line results in the threedimensional data presented here. This scan was taken in contact mode (see Ref. 233). In this linear gray scale image, white and black represent maximum positive and negative signal values, respectively.

certain gases on materials. Local probes have been used before for accurate measurement and sensitive detection of small quantities of gas molecules. In this realization, ${ }^{281-283}$ the hydrogen content in a Pd film was monitored via the change of the film resistivity and the bending of the film, mounted on a cantilever.

\section{B. Active samples}

The techniques presented in this article lend themselves to imaging of active samples in many ways. Pump and probe experiments are inherently high-speed techniques and used to resolve dynamic phenomena with ultrahigh temporal resolution. Standing electric fields can be imaged with many of the techniques discussed when used in collection mode, including miniature coaxial waveguides and resonant probes.

\section{Integrated circuits}

Most applications on active circuits to this point have been merely demonstrating the techniques involved. Consequently, many publications use open-ended waveguides or other simple structures where the electric field distribution is well known from theoretical considerations. One of the techniques suitable only for active samples is modulated scattering. It is clearly confined to map electric field distributions in integrated circuits at frequency ranges accessible by network analyzers. Modulated scattering has been applied to the mapping of tangential electric field components on microstrip antennas. ${ }^{199}$ Budka et al. have used modulated scattering to map electric fields above coupled line bandpass filters, ${ }^{200} \mathrm{a}$ distributed amplifier ${ }^{202}$ and a microstrip directional coupler. $^{201}$ For ultrafast sampling of pulses on waveguides, pump and probe experiments have been applied and mapped the spatio-temporal evolution of points, ${ }^{229} \operatorname{lines}^{233}$ or areas ${ }^{231}$ (Fig. 25). One of the most widely applied methods to probe active circuits is based on equivalent time sampling approaches. The previously discussed HFEFM uses frequency mixing on scanned probes and is entirely confined to imag-
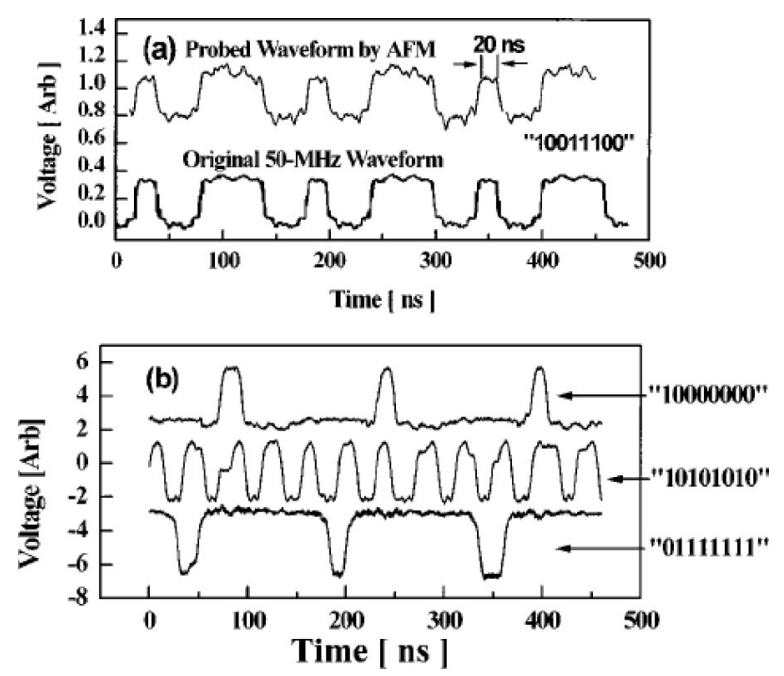

FIG. 26. (a) Time evolution of a digital test pattern at one point, sampled by HFEFM (top), compared to the pulses measured using a commercial sampling oscilloscope (below). (b) Different vector patterns as measured by the HFEFM sampler (see Ref. 208).

ing active samples. While many publications probe waveguides, ${ }^{219,220,226}$ others show measurement of microwave voltage amplitude on interdigital capacitors, ${ }^{216}$ traveling-wave amplifiers $^{214}$ and CMOS interconnect lines. ${ }^{212}$ Some publications have proven that digital pattern extraction is possible as well ${ }^{204,206,208,210,217}$ (Fig. 26). It is also possible to measure electric field components of standing microwaves using passive probes. Dutta et al. ${ }^{277}$ demon-
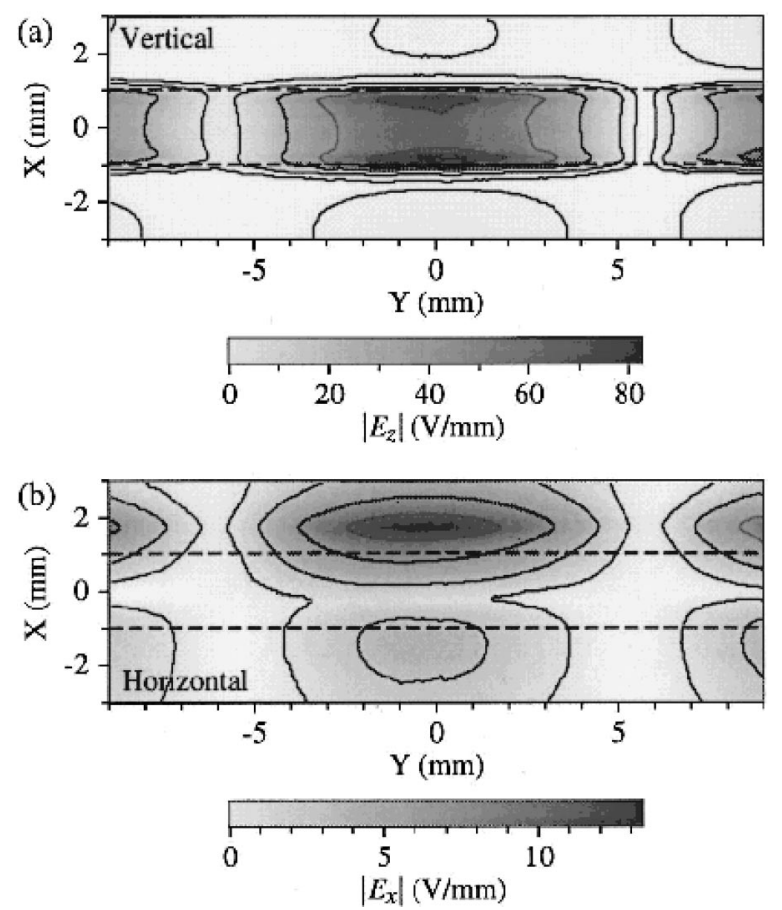

FIG. 27. Images of two components of electric field taken with the passive resonant coaxial line SNMM developed by Dutta et al. (see Ref. 277). The data are recorded above a 2-mm-wide copper microstrip driven at $8.05 \mathrm{GHz}$ and outlined by dashed lines. (a) Image with vertical probe $(\mathrm{h}=25 \mu \mathrm{m})$; contour lines at 70, 50, 30, 10 and $3 \mathrm{~V} / \mathrm{mm}$. (b) Image with horizontal probe $(\mathrm{h}=455 \mu \mathrm{m})$; contour lines at 11, 7.5, 4, and 1.5 V/mm. See Fig. 20 for the definition of $h$. 
strated this concept using a coaxial probe in different orientations to the DUT, connected to a resonant piece of coaxial cable (Fig. 27).

\section{Two-dimensional electron systems}

Localized spectroscopy of two-dimensional electron systems is of fundamental interest and may find applications in the field of solid-state quantum computing. The energies of interest lie in the very high-frequency microwave regime and in the far infrared. FIR radiation can strongly interact with these electrons and thus report on the system's structure and dynamics. Because of the relative inaccessibility of this frequency regime, only few publications exist. ${ }^{126-128}$ Keilmann et al. demonstrated in these articles the existence of a nonhomogeneous current distribution under the condition of a nonequilibrated population of edge states in a quantum Hall geometry.

\section{FUTURE DEVELOPMENTS}

This review surveyed the most common techniques used for high-frequency near-field microscopy. Some techniques not included in this review that are related to the topics discussed but not intrinsically made for high-frequency probing include dc electric force microscopy, ${ }^{284}$ single-electron transistor microscopy ${ }^{285}$ and IR interferometric charge detection. ${ }^{286}$

As the operating frequency and integration density of ICs increases and local material properties of semiconductor substrates become even more critical, it can be expected that local high-frequency probing techniques will grow in importance. The growing interaction between biological sciences and electrical engineering disciplines is further demonstrating that a wealth of biological information can be accessed via microwave measurements, which will further spur the growth of miniaturized microwave probes. It is a certainty that micro-electro-mechanical system developments will improve probe fabrication. As it becomes more common to produce micrometer sized three-dimensional structures, nearfield microwave probes will profit from this development through improved resolution and cheaper fabrication. For example, while coaxial structures are often employed in the microwave regime, their diameter is rarely in the micrometer range. By using carbon or silicon nanotubes as center conductors for an integrated probe, the resolution could be improved to $<10 \mathrm{~nm}$, with possible applications in SNOM. By better characterization and calibration of these local probes, connectorless microwave network analysis might also become a common practice. Ultimately, perfect lenses that can focus beyond the diffraction limit ${ }^{287,288}$ could revolutionize near-field microscopy, because they can achieve ultimate resolution a small distance away from the focusing element. For the foreseeable future, however, near-field microscopy of all wavelengths, employing the techniques discussed in this review, will grow as more and more researchers demand high-resolution and high-frequency imaging of active and passive systems.

\section{ACKNOWLEDGMENTS}

The authors acknowledge valuable discussions with Fritz Keilmann, Max-Planck-Institut für Biochemie in Martinsried, Germany. This work was supported by NSF (PECASE), ONR, and DARPA.

${ }^{1}$ E. Abbe, Arch. Mikrosc. Anat. Entwicklungsmech. 9, 413 (1873).

${ }^{2}$ E. Abbe, J. R. Microsc. Soc. 4, 348 (1884).

${ }^{3}$ E. H. Synge, Philos. Mag. 6, 356 (1928).

${ }^{4}$ E. H. Synge, Philos. Mag. 11, 65 (1931).

${ }^{5}$ H. A. Bethe, Phys. Rev. 66, 163 (1944).

${ }^{6}$ C. J. Bouwkamp, Philips Res. Rep. 5, 321 (1950).

${ }^{7}$ C. J. Bouwkamp, Philips Res. Rep. 5, 401 (1950).

${ }^{8}$ J. A. O'Keefe, J. Opt. Soc. Am. 46, 359 (1956).

${ }^{9}$ Z. Frait, Czech. J. Phys. 9, 403 (1959).

${ }^{10}$ R. F. Soohoo, J. Appl. Phys. 33, 1276 (1962).

${ }^{11}$ C. A. Bryant and J. B. Gunn, Rev. Sci. Instrum. 36, 1614 (1965).

${ }^{12}$ E. A. Ash and G. Nicholls, Nature (London) 237, 510 (1972).

${ }^{13}$ L. Novotny, B. Hecht, and D. Pohl, Ultramicroscopy 71, 341 (1998).

${ }^{14}$ G. Binnig and H. Rohrer, Helv. Phys. Acta 55, 726 (1982).

${ }^{15}$ U. C. Fischer, J. Vac. Sci. Technol. B 3, 386 (1984).

${ }^{16}$ A. Lewis, M. Isaacson, A. Harootunian, and A. Muray, Ultramicroscopy 13, 227 (1984).

${ }^{17}$ D. Pohl, W. Denk, and M. Lanz, Appl. Phys. Lett. 44, 651 (1984).

${ }^{18}$ U. Dürig, D. Pohl, and F. Rohner, J. Appl. Phys. 59, 3318 (1986).

${ }^{19}$ E. Betzig, A. Lewis, A. Harootunian M. Isaacson, and E. Kratschmer, Biophys. J. 49, 269 (1986).

${ }^{20}$ G. A. Massey, J. A. Davis, S. M. Katnik, and E. Omon, Appl. Opt. 24, 1498 (1985)

${ }^{21}$ M. Fee, S. Chu, and T. W. Hänsch, Opt. Commun. 69, 219 (1989).

${ }^{22}$ P. Kochanski, Phys. Rev. Lett. 62, 2285 (1989).

${ }^{23}$ G. Binnig, C. F. Quate, and C. Gerber, Phys. Rev. Lett. 56, 930 (1986).

${ }^{24}$ R. Toledo-Crow, P. C. Yang, Y. Chen, and M. Vaez-Iravani, Appl. Phys. Lett. 60, 2957 (1992).

${ }^{25}$ E. Betzig, P. L. Finn, and J. S. Weiner, Appl. Phys. Lett. 60, 2484 (1992).

${ }^{26}$ A. Harootunian, E. Betzig, M. Isaacson, and A. Lewis, Appl. Phys. Lett. 49, 674 (1986).

${ }^{27}$ E. Betzig, M. Isaacson, and A. Lewis, Appl. Phys. Lett. 51, 2088 (1987).

${ }^{28}$ K. Karrai and R. D. Grober, Ultramicroscopy 61, 197 (1995).

${ }^{29}$ K. Karrai and R. D. Grober, Appl. Phys. Lett. 66, 1842 (1995).

${ }^{30}$ P. Hoffmann, B. Dutoit, and R. P. Salathe, Ultramicroscopy 61, 165-170 (1995).

${ }^{31}$ T. Saiki, S. Mononobe, M. Ohtsu, N. Saito, and J. Kusano, Appl. Phys. Lett. 68, 2612 (1996).

${ }^{32}$ R. M. Stöckle, C. Fokas, V. Deckert, R. Zenobi, B. Sick, B. Hecht, and U. P. Wild, Appl. Phys. Lett. 75, 160 (1999).

${ }^{33}$ M. N. Islam, X. K. Zhao, A. A. Said, S. S. Mickel, and C. F. Vail, Appl. Phys. Lett. 71, 2886 (1997).

${ }^{34}$ N. Essaidi, Y. Chen, V. Kottler, E. Cambril, C. Mayeux, N. Ronarch, and C. Vieu, Appl. Opt. 37, 609 (1998).

${ }^{35}$ B. Hecht, B. Sick, U. P. Wild, V. Decker, R. Zenobi, O. J. F. Martin, and D. Pohl, J. Chem. Phys. 112, 7761 (2000).

${ }^{36}$ R. C. Dunn, Chem. Rev. 99, 2891 (1999).

${ }^{37}$ U. C. Fischer and D. W. Pohl, Phys. Rev. Lett. 62, 458 (1989).

${ }^{38}$ U. C. Fischer, in Scanning Tunneling Microscopy and Related Methods, edited by R. J. Behm, N. Garcia, and H. Rohrer (Kluwer, Dordrecht, 1990), Vol. 184, pp. 475-496.

${ }^{39}$ M. Specht, J. D. Pedarnig, W. M. Heckl, and Hänsch, Phys. Rev. Lett. 68, 476 (1992).

${ }^{40}$ German Patent No. DE 19522546 C2 by F. Keilmann and R. Guckenberger (19 February 1998).

${ }^{41}$ US Patent No. 5,789,742 by P. A. Wolff (4 August 1998).

${ }^{42}$ US Patent No. $5,770,885$ by U. C. Fischer (23 June 1998).

${ }^{43}$ J. Koglin, U. C. Fischer, and H. Fuchs, Phys. Rev. B 55, 7977 (1997).

${ }^{44}$ J. Ferber, U. C. Fischer, N. Hagedorn, and H. Fuchs, Appl. Phys. A: Mater. Sci. Process. 69, 581 (1999).

${ }^{45}$ J. Heimel, U. C. Fischer, and H. Fuchs, J. Microsc. 202, 53 (2001).

${ }^{46}$ J. Wessel, J. Opt. Soc. Am. B 2, 1538 (1985).

${ }^{47}$ F. Zenhausern, M. P. O'Boyle, and H. K. Wickramasinghe, Appl. Phys. Lett. 65, 1623 (1994).

${ }^{48}$ F. Zenhausern, Y. Martin, and H. K. Wickramasinghe, Science 269, 1083 (1995). 
${ }^{49}$ R. Bachelot, P. Gleyzes, and A. C. Boccara, Opt. Lett. 20, 1924 (1995).

${ }^{50}$ F. Keilmann, D. W. van der Weide, T. Eickelkamp, R. Merz, and D. Stöckle, Opt. Commun. 129, 15 (1996).

${ }^{51}$ M. Labardi, S. Patane, and M. Allegrini, Appl. Phys. Lett. 77, 621 (2000).

${ }^{52}$ B. Knoll and F. Keilmann, Opt. Commun. 182, 321 (2000).

${ }^{53}$ R. Hillenbrand and F. Keilmann, Phys. Rev. Lett. 85, 3029 (2000).

${ }^{54}$ R. Hillenbrand, B. Knoll, and F. Keilmann, J. Microsc. 202, 77 (2001).

${ }^{55}$ H. U. Danzebrink, T. Dziomba, T. Sulzbach, O. Ohlsson, C. Lehrer, and L. Frey, J. Microsc. 194, 335 (1999).

${ }^{56}$ A. G. T. Ruiter, M. H. P. Moers, A. Jalocha, and N. F. van Hulst, Ultramicroscopy 61, 139 (1995).

${ }^{57}$ A. G. T. Ruiter, M. H. P. Moers, N. F. van Hulst, and M. de Boer, J. Vac. Sci. Technol. B 14, 597 (1996).

${ }^{58}$ H. Zhou, A. Midha, G. Mills, L. Donaldson, and J. M. R. Weaver, Appl. Phys. Lett. 75, 1824 (1999).

${ }^{59}$ P. N. Minh, T. Ono, and M. Esashi, Sens. Actuators A 80, 163 (2000).

${ }^{60}$ D. Drews et al., Nanotechnology 10, 61 (1999).

${ }^{61}$ W. Noell, M. Abraham, K. Mayr, A. Ruf, J. Barenz, O. Hollricher, O. Marti, and P. Güthner, Appl. Phys. Lett. 70, 1236 (1997).

${ }^{62}$ S. Werner, O. Rudow, C. Mihalcae, and E. Osterschulze, Appl. Phys. Lett. 66, S367 (1998).

${ }^{63}$ B. Belier, A. Santoso, J. Bonnafe, L. Nicu, P. Temple-boyer, and C. Bergaud, Appl. Phys. Lett. 77, 1768 (2000).

${ }^{64}$ K. Karrai, X. Lorenz, and L. Novotny, Appl. Phys. Lett. 77, 3459 (2000).

${ }^{65}$ D. A. Fletcher, K. B. Crozier, C. F. Quate, C. F. Quate, G. S. Kino, K. E. goodson, D. Simanovskii, and D. V. Palanker, Appl. Phys. Lett. 78, 3589 (2001).

${ }^{66}$ D. A. Fletcher, K. B. Crozier, K. W. Guarini, S. C. Minne, G. S. Kino, C. F. Quate, and K. E. Goodson, J. Microelectromech. Syst. 10, 450 (2001).

${ }^{67}$ S. Heisig and E. Oesterschulze, Appl. Phys. A: Mater. Sci. Process. 66, S385 (1998).

${ }^{68}$ S. Heisig, O. Rudow, and E. Oesterschulze, J. Vac. Sci. Technol. B 18, 1134 (2000).

${ }^{69}$ H. U. Danzebrink, O. Ohlsson, and G. Wilkening, Ultramicroscopy 61, 131 (1995).

${ }^{70}$ H. U. Danzebrink, G. Wilkening, and O. Ohlsson, Appl. Phys. Lett. 67, 1981 (1995)

${ }^{71}$ S. Akamine, H. Kuwano, and H. Yamada, Appl. Phys. Lett. 68, 579 (1996).

${ }^{72}$ Y. Tanaka, K. Fukuzawa, and H. Kuwano, J. Appl. Phys. 83, 3547 (1998).

${ }^{73}$ R. C. Davis, C. C. Williams, and P. Neuzil, Appl. Phys. Lett. 66, 2309 (1995).

${ }^{74}$ R. C. Davis, C. C. Williams, and P. Neuzil, Opt. Lett. 21, 447 (1996).

${ }^{75}$ R. C. Davis and C. C. Williams, Appl. Phys. Lett. 69, 1179 (1996).

${ }^{76}$ T. Leinhos, M. Stopka, and E. Oesterschulze, Appl. Phys. A: Mater. Sci. Process. 66, S65 (1998).

${ }^{77}$ G. Schürmann, W. Noell, U. Staufer, and N. F. de Rooij, Ultramicroscopy 82, 33 (2000).

${ }^{78}$ B. Rosner, T. Bork, V. Agrawal, P. Neuzil, and D. W. van der Weide, Proc. SPIE 4456, 127 (2001).

${ }^{79}$ B. Rosner, T. Bork, V. Agrawal, and D. W. van der Weide, Sens. Actuators A (in press).

${ }^{80}$ D. Courjon, K. Sarayeddine, and M. Spajer, Opt. Commun. 71, 23 (1989).

${ }^{81}$ D. DeFornel, E. Lesniewska, L. Salomon, and J. P. Goudonnet, Opt. Commun. 102, 1 (1993).

${ }^{82}$ A. Piednoir, F. Creuzet, C. Licoppe, and J. M. Ortega, Ultramicroscopy 57, 282 (1995).

${ }^{83}$ T. Nakano and S. Kawata, Optik (Stuttgart) 94, 159 (1993).

${ }^{84}$ A. G. Jeung, S. Erramilli, M. K. Hong, P. Huie, and T. I. Smith, Proc. SPIE 3153, 117 (1997).

${ }^{85}$ A. Cricenti, R. Generosi, P. Perfetti, J. M. Gilligan, N. H. Tolk, C. Coluzza, and G. Margaritondo, Appl. Phys. Lett. 73, 151 (1998).

${ }^{86}$ D. T. Schaafsma, R. Mossadegh, J. S. Sanghera, I. D. Aggarwal, J. M. Gilligan, N. H. Tolk, M. Luce, R. Generosi, and P. Perfetti, Ultramicroscopy 77, 77 (1999).

${ }^{87}$ D. T. Schaafsma, R. Mossadegh, J. S. Sanghera, I. D. Aggarwal, M. Luce, R. Generosi, P. Pefetti, A. Cricenti, and J. M. Gilligan, Opt. Eng. (Bellingham) 38, 1381 (1999).

${ }^{88}$ B. Dragnea, J. Preusser, W. Schade, S. R. Leone, and W. D. Hinsberg, J. Appl. Phys. 86, 2795 (1999).

${ }^{89}$ C. A. Michaels, S. J. Stranick, L. J. Richter, and R. R. Cavanagh, J. Appl. Phys. 88, 4832 (2000).

${ }^{90}$ J. S. Sanghera, L. B. Shaw, D. B. Talley, L. E. Busse, and I. D. Aggarwal, Proc. SPIE 3907, 461 (2000).
${ }^{91}$ D. B. Talley, L. B. Shaw, J. S. Sanghera, I. D. Aggarwal, A. Cricenti, R. Generosi, M. Luce, G. Margaritondo, and J. M. Gilligan, Mater. Lett. 42, 339 (2000).

${ }^{92}$ B. Dragnea, J. Preusser, J. M. Szarko, S. R. Leone, and W. D. Hinsberg, J. Vac. Sci. Technol. B 19, 142 (2001).

${ }^{93}$ B. Knoll and F. Keilmann, Appl. Phys. A: Mater. Sci. Process. 66, 477 (1998).

${ }^{94}$ B. Knoll and F. Keilmann, Nature (London) 399, 134 (1999).

${ }^{95}$ A. Lahrech, R. Bachelot, P. Gleyzes, and A. C. Boccara, Opt. Lett. 21, 1315 (1996)

${ }^{96}$ D. V. Palanker, G. M. H. Knippels, T. I. Smith, and H. A. Schwettman, Opt. Commun. 148, 215 (1998).

${ }^{97}$ A. Hammiche, H. M. Pollock, M. Reading, M. Claybourn, P. Turner, and K. Jewkes, Appl. Spectrosc. 53, 810 (1999).

${ }^{98}$ U.S. Patent No. 5,696,372 by R. D. Grober (9 December 1997).

${ }^{99}$ R. D. Grober, R. J. Schoelkopf, and D. E. Prober, Appl. Phys. Lett. 70, 1354 (1997).

${ }^{100}$ D. W. Pohl, in Near-Field Optics: Principles and Applications/The Second Asia-Pacific Workshop on Near Field Optics, Beijing, China October 20-23, 1999, edited by M. Ohtsu and X. Zhu (World Scientific, Singapore, 2000).

${ }^{101}$ B. Rosner, J. Peck, and D. W. van der Weide, IEEE Trans. Antennas Propag. (in press).

${ }^{102}$ V. Daneu, D. Sokoloff, A. Sanchez, and A. Javan, Appl. Phys. Lett. 15, 398 (1969).

${ }^{103}$ L. M. Matarrese and K. M. Evenson, Appl. Phys. Lett. 17, 8 (1970).

${ }^{104}$ S. E. Schwarz and B. T. Ulrich, J. Appl. Phys. 48, 1870 (1977).

${ }^{105}$ D. B. Rutledge, S. E. Schwarz, and A. T. Adams, Infrared Phys. 18, 713 (1978).

${ }^{106}$ S. Wang, Appl. Phys. Lett. 28, 303 (1976).

${ }^{107}$ W. Krieger, T. Suzuki, and M. Völcker, Phys. Rev. B 41, 10229 (1990).

${ }^{108}$ M. Völcker, W. Krieger, T. Suzuki, and H. Walther, J. Vac. Sci. Technol. B 9, 541 (1991).

${ }^{109}$ M. Völcker, W. Krieger, and H. Walther, J. Vac. Sci. Technol. B 12, 2129 (1994).

${ }^{110}$ I. Wilke, W. Herrmann, and F. K. Kneubühl, Appl. Phys. B: Lasers Opt. 58, 87 (1994).

${ }^{111}$ L. Wilke, Y. Oppliger, W. Herrmann, and F. K. Kneubühl, Appl. Phys. B: Lasers Opt. 58, 329 (1994).

${ }^{112}$ E. N. Grossman, IEEE International Symposium on Electromagnetic Compatibility, Symposium Record 102-107 (1995).

${ }^{113}$ C. Fumeaux, W. Herrmann, F. K. Kneubühl, H. Rothuizen, B. Lipphardt, and C. O. Weiss, Appl. Phys. B: Lasers Opt. 66, 327 (1998).

${ }^{114}$ B. Dragnea and S. R. Leone, Int. Rev. Phys. Chem. 20, 59 (2001).

${ }^{115}$ H. M. Pollock and D. A. Smith, in Handbook of Vibrational Spectroscopy, edited by J. M. Chalmers and P. R. Griffiths (Wiley, New York, 2001.

${ }^{116}$ S. Hunsche, M. Koch, I. Brener, and M. C. Nuss, Opt. Commun. 150, 22 (1998).

${ }^{117}$ D. W. van der Weide, J. S. Bostak, B. A. Auld, and D. M. Bloom, Appl. Phys. Lett. 62, 22 (1993).

${ }^{118}$ D. W. van der Weide, J. Opt. Soc. Am. B 11, 2553 (1994).

${ }^{119}$ U.S. Patent No. $5,748,309$ by D. W. van der Weide and F. Keilmann (5 May 1998)

${ }^{120}$ J. Faist, F. Capasso, C. Sirtori, D. L. Sivco, A. L. Hutchinson, and A. Y. Cho, Nature (London) 387, 777 (1997).

${ }^{121}$ G. Scamarcio, F. Capasso, C. Sirtori, J. Faist, A. L. Hutchinson, D. L. Sivco, and A. Y. Cho, Science 276, 773 (1997).

${ }^{122}$ C. Gmachl, F. Capasso, E. E. Narimanov, J. U. Nöckel, A. D. Stone, J. Faist, D. L. Sivco, and A. Y. Cho, Science 280, 1493 (1998).

${ }^{123}$ C. Sirtori, C. Gmachl, F. Capasso, J. Faist, D. L. Sivco, A. L. Hutchinson, and A. Y. Cho, Opt. Lett. 23, 1366 (1998).

${ }^{124}$ K. Namjou, S. Cai, E. A. Whittaker, J. Faist, C. Gamachl, F. Capasso, D. L. Sivco, and A. Y. Cho, Opt. Lett. 23, 219 (1998).

${ }^{125}$ G. A. Massey, Appl. Opt. 23, 658 (1984).

${ }^{126}$ F. Keilmann and R. Merz, in Near Field Optics, edited by D. W. Pohl and D. Courjon (Kluwer, Doredrecht, 1993), pp. 317-324.

${ }^{127}$ R. Merz, F. Keilmann, R. J. Haug, and K. Ploog, Phys. Rev. Lett. 70, 651 (1993).

${ }^{128}$ F. Keilmann, Infrared Phys. Technol. 36, 217 (1995).

${ }^{129}$ U.S. Patent No. $4,994,818$ by F. Keilmann (19 February 1991).

${ }^{130}$ U. C. Fischer and M. Zapletal, Ultramicroscopy 42-44, 393 (1992). 
${ }^{131}$ C. W. McCutchen, Scanning 17, 15 (1995).

${ }^{132}$ B. Knoll and F. Keilmann, Appl. Phys. Lett. 77, 3980 (2000).

${ }^{133}$ L. Novotny, D. W. Pohl, and P. Regli, Ultramicroscopy 57, 180 (1995).

${ }^{134}$ D. W. Pohl, L. Novotny, B. Hecht, and H. Heinzelmann, Thin Solid Films 273, 161 (1996)

${ }^{135}$ H. Furukawa and S. Kawata, Opt. Commun. 148, 221 (1998).

${ }^{136}$ P. M. Adam, P. Royer, R. Laddada, and J. L. Bijeon, Appl. Opt. 37, 1814 (1998).

${ }^{137}$ P. M. Adam, J. L. Bijeon, G. Viardot, and P. Royer, Opt. Commun. 174, 91 (2000).

${ }^{138}$ I. Sh. Averbukh, B. M. Chernobrod, O. A. Sedletsky, and Y. Prior, Opt. Commun. 174, 33 (2000).

${ }^{139}$ R. E. Larsen and H. Metiu, J. Chem. Phys. 114, 6851 (2001).

${ }^{140}$ H. Cory, A. C. Boccara, J. C. Rivoal, and A. Lahrech, Microwave Opt. Technol. Lett. 18, 120 (1998).

${ }^{141}$ O. Mitrofanov, I. Brener, M. C. Wanke, R. R. Ruel, J. D. Wynn, and A. J. Bruce, Appl. Phys. Lett. 77, 591 (2000).

${ }^{142}$ L. Bozec, A. Hammiche, H. M. Pollock, M. Conroy, J. M. Chalmers, N. J. Everall, and L. Turin, J. Appl. Phys. 90, 5159 (2001).

${ }^{143}$ E. Wolfgang, Microelectron. Eng. 4, 77 (1986).

${ }^{144}$ J. Bokor, A. M. Johnson, R. H. Storz, and W. M. Simpson, Appl. Phys. Lett. 49, 226 (1986).

${ }^{145}$ K. J. Weingarten, M. J. W. Rodwell, and D. M. Bloom, IEEE J. Quantum Electron. 24, 198 (1988)

${ }^{146}$ W. Mertin, C. Böhm, L. J. Balk, and E. Kubalek, IEEE MTT-S Int. Microwave Symp. Dig. 3, 1597 (1994).

${ }^{147}$ G. David, W. Schroeder, D. Jäger, and I. Wolff, IEEE MTT-S Int. Microwave Symp. Dig. 3, 1049 (1995).

${ }^{148}$ Z. Frait, Czech. J. Phys. 7, 232 (1957).

${ }^{149}$ M. C. Decreton and F. E. Gardiol, IEEE Trans. Instrum. Meas. IM23, 434 (1974).

${ }^{150}$ B. Knoll and F. Keilmann, Opt. Commun. 162, 177 (1999).

${ }^{151}$ J. R. Mosig, J. C. E. Besson, M. Gex-Fabry, and F. E. Gardiol, IEEE Trans. Instrum. Meas. IM30, 46 (1981).

${ }^{152}$ G. B. Gajda and S. S. Stuchly, IEEE Trans. Microwave Theory Tech. MTT31, 380 (1983).

${ }^{153}$ G. Q. Jiang, W. H. Wong, E. Y. Raskovich, and W. G. Clark, Rev. Sci. Instrum. 64, 1622 (1993).

${ }^{154}$ G. Q. Jiang, W. H. Wong, E. Y. Raskovich, and W. G. Clark, Rev. Sci. Instrum. 64, 1614 (1993).

${ }^{155}$ C. L. Pournaropoulos and D. Misra, IEEE Trans. Instrum. Meas. 43, 111 (1994).

${ }^{156}$ C. L. Li and K. M. Chen, IEEE Trans. Instrum. Meas. 44, 19 (1995).

${ }^{157}$ B. G. Colpitts, IEEE Trans. Microwave Theory Tech. 44, 160 (1996).

${ }^{158}$ Z. M. Xie, E. K. N. Yung, and R. S. Chen, Microwave Opt. Technol. Lett. 18, 95 (1998).

${ }^{159}$ Y. Xu, F. M. Ghannouchi, and R. G. Bosisio, IEEE Trans. Microwave Theory Tech. 40, 143 (1992).

${ }^{160}$ D. Bérubé, F. M. Ghannouchi, and P. Savard, IEEE Trans. Microwave Theory Tech. 44, 1928 (1996).

${ }^{161}$ S. Wang, M. Niu, and D. Xu, IEEE Trans. Microwave Theory Tech. 46, 2145 (1998)

${ }^{162}$ W. Hu, A. Thanawalla, B. J. Feenstra, F. C. Wellstood, and S. M. Anlage, Appl. Phys. Lett. 75, 2824 (1999).

${ }^{163}$ D. W. van der Weide and P. Neuzil, J. Vac. Sci. Technol. B 14, 4144 (1996).

${ }^{164}$ D. K. Misra and D. Eungdamrong, Circuits and Systems ISCAS, 2001 (unpublished).

${ }^{165}$ Y. Gao and I. Wolff, IEEE Trans. Microwave Theory Tech. 46, 907 (1998).

${ }^{166}$ Y. Gao and I. Wolff, IEEE Trans. Microwave Theory Tech. 44, 911 (1996).

${ }^{167}$ S. S. Osofsky and S. E. Schwarz, IEEE Trans. Microwave Theory Tech. 40, 1701 (1992).

${ }^{168}$ M. Kanda, IEEE Trans. Electromagn. Compat. 26, 102 (1994).

${ }^{169}$ D. W. van der Weide, Appl. Phys. Lett. 70, 677 (1997).

${ }^{170}$ S. Heisig, H. U. Danzebrink, A. Leyk, W. Mertin, S. Münster, and E. Oesterschulze, Ultramicroscopy 71, 99 (1998).

${ }^{171}$ E. Oesterschulze, Appl. Phys. A: Mater. Sci. Process. 66, S3 (1998).

${ }^{172}$ A. Kramer, F. Keilmann, B. Knoll, and R. Guckenberger, Micron 27, 413 (1996).

${ }^{173}$ B. Michel, W. Mizutani, R. Schierle, A. Jarosch, W. Knop, H. Benedickter, W. Bächthold, and H. Rohrer, Rev. Sci. Instrum. 63, 4080 (1992).

${ }^{174}$ S. J. Stranick and P. S. Weiss, Rev. Sci. Instrum. 64, 1232 (1993).
${ }^{175}$ S. J. Stranick and P. S. Weiss, Rev. Sci. Instrum. 65, 918 (1994).

${ }^{176}$ S. J. Stranick and P. S. Weiss, J. Phys. Chem. 98, 1762 (1994).

${ }^{177}$ S. J. Stranick, M. M. Kamna, and P. S. Weiss, Rev. Sci. Instrum. 65, 3211 (1994).

${ }^{178}$ S. J. Stranick, L. A. Bumm, M. M. Kamna, and P. S. Weiss, in Photons and Local Probes, edited by O. Marti and R. Möller (Kluwer, Dordrecht, 1995), pp. 221-233.

${ }^{179}$ L. A. Bumm and P. S. Weiss, Rev. Sci. Instrum. 66, 4140 (1995).

${ }^{180}$ L. A. Bumm, J. J. Arnold, M. T. Cygan, T. d. Dunbar, T. P. Burgin, L. Jones II, D. L. Allara, J. M. Tour, and P. S. Weiss, Science 271, 1705 (1996).

${ }^{181}$ G. S. McCarty and P. S. Weiss, Chem. Rev. 99, 1983 (1999).

${ }^{182}$ A. V. Bragas, S. M. Landi, and O. E. Martínez, Appl. Phys. Lett. 72, 2075 (1998).

${ }^{183}$ A. V. Zayats and V. Sandoghdar, Opt. Commun. 178, 245 (2000).

${ }^{184}$ J. P. Bourgoin, M. B. Johnson, and B. Michel, Appl. Phys. Lett. 65, 2045 (1994).

${ }^{185}$ M. B. Johnson, J. P. Bourgoin, and B. Michel, Microelectron. Eng. 27, 539 (1995).

${ }^{186}$ W. Siefert, E. Gerner, M. Stachel, and K. Dransfeld, Ultramicroscopy 42-44, 379 (1992)

${ }^{187}$ C. C. Williams, J. Slinkman, W. P. Hough, and H. K. Wickramasinghe, Appl. Phys. Lett. 55, 1662 (1989).

${ }^{188}$ R. C. Barrett and C. F. Quate, J. Appl. Phys. 70, 2725 (1991).

${ }^{189}$ K. Goto and K. Hane, Rev. Sci. Instrum. 68, 120 (1996).

${ }^{190}$ Y. Huang, C. C. Williams, and J. Slinkman, Appl. Phys. Lett. 66, 344 (1995).

${ }^{191}$ S. Lanyi, J. Török, and P. Rehurek, Rev. Sci. Instrum. 65, 2258 (1994).

${ }^{192}$ S. Lanyi and J. Török, J. Electr. Eng. 46, 126 (1995).

${ }^{193}$ S. Lanyi, J. Török, and P. Rehurek, J. Vac. Sci. Technol. B 14, 892 (1996).

${ }^{194}$ A. Lewis, E. Shambrot, A. Radko, K. Lieberman, S. Ezekiel, D. Veinger, and G. Yampolski, Proc. IEEE 88, 1471 (2000).

${ }^{195}$ F. Keilmann, B. Knoll, and A. Kramer, Phys. Status Solidi B 215, 849 (1999).

${ }^{196}$ J. H. Richmond, Inst. Radio Eng. Trans. MTT3, 13 (1955).

${ }^{197}$ A. L. Cullen and J. C. Parr, Proc. IEE B 102, 836 (1955).

${ }^{198}$ R. Justice and V. H. Rumsey, Inst. Radio Eng. Trans. AP3, 177 (1955).

${ }^{199}$ S. A. Bokhari, J. F. Zürcher, J. R. Mosig, and R. E. Gardiol, IEEE Trans. Antennas Propag. 43, 188 (1995).

${ }^{200}$ T. P. Budka, E. M. Tentzeris, S. D. Waclawik, N. I. Dib, L. P. B. Katehi, and G. M. Rebiez, IEEE MTT-S Int. Microwave Symp. Dig. 3, 1487 (1995).

${ }^{201}$ T. P. Budka, S. D. Waclawik, and G. M. Rebeiz, IEEE Trans. Microwave Theory Tech. 44, 2174 (1996)

${ }^{202}$ T. P. Budka, S. D. Waclawik, and G. M. Rebeiz, IEEE MTT-S Int. Microwave Symp. Dig. 3, 1703 (1996).

${ }^{203}$ T. P. Budka, E. M. Tentzeris, S. D. Waclawik, N. I. Dib, L. P. B. Katehi, and G. M. Rebeiz, Microwave J. (Euro ed.) 41, 94 (1998).

${ }^{204}$ B. A. Nechay, F. Ho, A. S. Hou, and D. M. Bloom, J. Vac. Sci. Technol. B 13, 1369 (1995).

${ }^{205}$ A. S. Hou, F. Ho, and D. M. Bloom, Electron. Lett. 28, 2302 (1992).

${ }^{206}$ F. Ho, A. S. Hou, and D. M. Bloom, Electron. Lett. 30, 560 (1994).

${ }^{207}$ F. Ho, A. S. Hou, B. A. Nechay, and D. M. Bloom, Ultrafast Electron. Optoelectron. 13, 166 (1995).

${ }^{208}$ J. W. Hong, Z. G. Kim, A. S. Hou, and S. Park, Rev. Sci. Instrum. 68, 4506 (1997).

${ }^{209}$ G. E. Bridges, R. A. Said, and D. J. Thompson, Electron. Lett. 29, 1447 (1993).

${ }^{210}$ G. E. Bridges, R. A. Said, M. Mittal, and D. J. Thompson, J. Vac. Sci. Technol. B 13, 1375 (1995).

${ }^{211}$ G. E. Bridges, Electron. Lett. 35, 1724 (1999).

${ }^{212}$ R. A. Said, S. P. Cheung, and G. E. Bridges, J. Vac. Sci. Technol. B 18, 626 (2000).

${ }^{213}$ C. Böhm, F. Saurenbach, P. Taschner, C. Roths, and E. Kubalek, J. Phys. D 26, 1801 (1993).

${ }^{214}$ C. Böhm, C. Roths, and E. Kubalek, Electron. Lett. 24, 91 (1994).

${ }^{215}$ A. Leyk, C. Böhm, D. W. van der Weide, and E. Kubalek, Electron. Lett. 31, 1046 (1995).

${ }^{216}$ A. Leyk and E. Kubalek, Microelectron. Eng. 31, 187 (1996).

${ }^{217}$ J. Sprengepiel, C. Böhm, and E. Kubalek, Microelectron. Eng. 31, 181 (1996).

${ }^{218}$ C. Böhm, M. Otterbeck, S. Lipp, L. Frey, R. Reuter, A. Leyk, W. Mertin, 
F. J. Tegude, and E. Kubalek, IEEE MTT-S Int. Microwave Symp. Dig. 3, 1529 (1996).

${ }^{219}$ C. Böhm, J. Sprengepiel, M. Otterbeck, and E. Kubalek, J. Vac. Sci. Technol. B 14, 842 (1996).

${ }^{220}$ C. Böhm, Microelectron. Eng. 31, 171 (1996).

${ }^{221}$ D. W. van der Weide, Appl. Phys. Lett. 65, 881 (1994).

${ }^{222}$ C. Schönenberger and S. F. Alvarado, Phys. Rev. Lett. 65, 3162 (1990).

${ }^{223}$ S. Weiss, D. F. Ogletree, D. Botkin, M. Salmeron, and D. S. Chemla, Appl. Phys. Lett. 63, 2567 (1993).

${ }^{224}$ D. Botkin, J. Glass, D. S. Chemla, D. F. Ogletree, M. Salmeron, and S. Weiss, Appl. Phys. Lett. 69, 1321 (1996).

${ }^{225}$ G. Nunes and M. R. Freeman, Science 262, 1029 (1993).

${ }^{226}$ K. Takeuchi and Y. Kasahara, Appl. Phys. Lett. 63, 3548 (1993).

${ }^{227}$ K. Takeuchi, A. Mizuhara, and Y. Kasahara, IEEE Trans. Instrum. Meas. 44, 815 (1995).

${ }^{228}$ K. Takeuchi and A. Mizuhara, Electron. Lett. 32, 1709 (1996).

${ }^{229}$ J. Kim, S. Williamson, J. Nees, S. Wakana, and J. Whitaker, Appl. Phys. Lett. 62, 2268 (1993)

${ }^{230}$ J. Nees, D. Craig, S. Hama, and S. Wakana, Ultrafast Electronics and Optoelectronics.

${ }^{231}$ K. Takeuchi and A. Mizuhara, IEEE MTT-S Int. Microwave Symp. Dig. 3, 643 (1997).

${ }^{232}$ W. M. Steffens, S. Heisig, U. D. Keil, and E. Oesterschulze, Appl. Phys. B: Lasers Opt. 69, 455 (1999).

${ }^{233}$ E. Oesterschulze, S. Heisig, and W. M. Steffens, J. Vac. Sci. Technol. B 19, 107 (2001).

${ }^{234}$ T. Pfeifer, H. M. Heiliger, H. G. Roskos, and H. Kurz, in Ref. 207.

${ }^{235}$ T. Pfeifer, H.-M. Heiliger, E. Stein von Kamienski, H. G. Roskos, and H. Kurz, J. Opt. Soc. Am. B 11, 2547 (1994).

${ }^{236}$ T. Löffler, T. Pfeifer, H. G. Roskos, H. Kurz, and D. W. van der Weide, Microelectron. Eng. 31, 397 (1996).

${ }^{237}$ T. Pfeifer, T. Löffler, H. G. Roskos, H. Kurz, M. Singer, and E. M. Biebl, Electron. Lett. 32, 1305 (1996).

${ }^{238}$ P. Gruetter, H. J. Mamin, and D. Rugar, in Scanning Tunneling Microscopy II. Further Applications and Related Scanning Techniques (Springer, Berlin, 1995), pp. 151-207.

${ }^{239}$ V. Agrawal, P. Neuzil, and D. W. van der Weide, Appl. Phys. Lett. 71, 2343 (1997).

${ }^{240}$ S. C. Lee, C. P. Vlahacos, B. J. Feenstra, A. Schwartz, D. E. Steinhauer, F. C. Wellstood, and S. M. Anlage, Appl. Phys. Lett. 77, 4404 (2000).

${ }^{241}$ P. Johansson, S. P. Apell, and D. R. Penn, Phys. Rev. B 64, 054411/1-13 (2001).

${ }^{242}$ J. R. Kirtley, M. B. Ketchen, K. G. Stawiasz, J. Z. Sun, W. J. Gallagher, S. H. Blanton, and S. J. Wind, Appl. Phys. Lett. 66, 1138 (1995).

${ }^{243}$ F. C. Wellstood, Y. Gim, A. Amar, R. C. Black, and A. Mathai, IEEE Trans. Appl. Supercond. 7, 3134 (1997).

${ }^{244}$ R. C. Black, F. C. Wellstood, E. Dantsker, A. H. Miklich, J. J. Kingston, D. T. Nemeth, and J. Clarke, Appl. Phys. Lett. 64, 100 (1994).

${ }^{245}$ R. C. Black, F. C. Wellstood, E. Dantsker, A. H. Miklich, D. Koelle, F. Ludwig, and J. Clarke, IEEE Trans. Appl. Supercond. 5, 2137, (1995).

${ }^{246}$ R. C. Black, F. C. Wellstood, E. Dantsker, A. H. Miklich, D. Koelle, F. Ludwig, and J. Clarke, Appl. Phys. Lett. 66, 1267 (1995).

${ }^{247}$ R. C. Black, F. C. Wellstood, E. Dantsker, A. H. Miklich, D. T. Nemeth, D. Koelle, F. Ludwig, and J. Clarke, Appl. Phys. Lett. 66, 99 (1995).

${ }^{248}$ M. Golosovsky and D. Davidov, Appl. Phys. Lett. 68, 1579 (1996).

${ }^{249}$ A. Mohammed-Djafari, N. Qaddoum, and R. Zoughi, Proc. SPIE 3816, 274 (1999).

${ }^{250}$ M. Golosovsky, A. Galkin, and D. Davidov, IEEE Trans. Microwave Theory Tech. 44, 1390 (1996).

${ }^{251}$ A. F. Lann, M. Golosovsky, and D. Davidov, Appl. Phys. Lett. 75, 603 (1999).

${ }^{252}$ A. F. Lann, M. Golosovsky, and D. Davidov, Appl. Phys. Lett. 73, 2832 (1998).

${ }^{253}$ M. Abu-Teir, M. Golosovsky, D. Davidov, A. Frenkel, and H. Goldberger, Rev. Sci. Instrum. 72, 2073 (2001).
${ }^{254}$ J. Bae, T. Okamoto, T. Fujii, K. Mizuno, and T. Nozokido, Appl. Phys. Lett. 71, 3581 (1997).

${ }^{255}$ T. Nozokido, J. Bae, and K. Mizuno, IEEE Trans. Microwave Theory Tech. 49, 491 (2001).

${ }^{256}$ M. Tabib-Azar, N. S. Shoemaker, and S. Harris, Meas. Sci. Technol. 4, 583 (1993).

${ }^{257}$ M. Tabib-Azar, D. P. Su, A. Pohar, S. r. LeClair, and G. Ponchak, Rev. Sci. Instrum. 70, 1725 (1999).

${ }^{258}$ M. Tabib-Azar, P. S. Pathak, G. Ponchak, and S. R. LeClari, Rev. Sci. Instrum. 70, 2783 (1999).

${ }^{259}$ M. Tabib-Azar and D. Akinwande, Rev. Sci. Instrum. 71, 1460 (2000).

${ }^{260}$ M. Tabib-Azar, D. Akinwande, G. E. Ponchak, and S. R. LeClair, Rev. Sci. Instrum. 70, 3083 (1999).

${ }^{261}$ Y. Cho, A. Kirihara, and T. Saeki, Rev. Sci. Instrum. 67, 2297 (1996).

${ }^{262}$ T. Wei, X.-D. Xiang, W. G. Wallace-Freedman, and P. G. Schultz, Appl. Phys. Lett. 68, 3506 (1996).

${ }^{263}$ C. Gao, T. Wei, F. Duewer, Y. Lu, and S. D. Xiang, Appl. Phys. Lett. 71, 1872 (1997).

${ }^{264}$ Y. Lu, T. Wei, F. Duewer, Y. Lu, N. B. Ming, P. G. Schultz, and X. D. Xiang, Science 276, 2004 (1997).

${ }^{265}$ C. Gao, F. Duewer, Y. Lu, and X. D. Xiang, Appl. Phys. Lett. 73, 1146 (1998).

${ }^{266}$ I. Takeuchi et al., Appl. Phys. Lett. 71, 2026 (1997).

${ }^{267}$ C. Gao and X. D. Xiang, Rev. Sci. Instrum. 69, 3846 (1998).

${ }^{268}$ C. Gao, F. Duewer, and X. D. Xiang, Appl. Phys. Lett. 75, 3005 (1999).

${ }^{269}$ F. Duewer, C. Gao, I. Takeuchi, and X. D. Xiang, Appl. Phys. Lett. 74, 2696 (1999).

${ }^{270}$ C. P. Vlahacos, R. C. Black, S. M. Anlage, A. Amar, and F. C. Wellstood, Appl. Phys. Lett. 69, 3272 (1996).

${ }^{271}$ S. M. Anlage, C. P. Vlahacos, S. Dutta, and F. C. Wellstood, IEEE Trans. Appl. Superond. 7, 3686 (1997).

${ }^{272}$ D. E. Steinhauer, C. P. Vlahacos, S. Dutta, F. C. Wellstood, and S. M. Anlage, Appl. Phys. Lett. 71, 1736 (1997).

${ }^{273}$ C. P. Vlahacos, D. E. Steinhauer, S. Dutta, B. J. Feenstra, S. M. Anlage, and F. C. Wellstood, Appl. Phys. Lett. 72, 1778 (1998).

${ }^{274}$ S. M. Anlage, D. E. Steinhauer, C. P. Vlahacos, B. J. Feenstra, A. Thanawalla, W. Hu, S. Dutta, and F. C. Wellstood, IEEE Trans. Appl. Supercond. 9, 4127 (1999).

${ }^{275}$ D. E. Steinhauer, C. P. Vlahacos, F. C. Wellstood, S. M. Anlage, C. Canedy, R. Ramesh, A. Stanishevsky, and J. Melngailis, Appl. Phys. Lett. 75, 3180 (1999).

${ }^{276}$ C. P. Vlahacos, D. E. Steinhauer, S. Dutta, B. J. Feenstra, S. M. Anlage, and F. C. Wellstood, Microsc. Anal. (accepted).

${ }^{277}$ S. K. Dutta, C. P. Vlahacos, D. E. Steinhauer, A. S. Thanawalla, B. J. Feenstra, F. C. Wellstood, and S. M. Anlage, Appl. Phys. Lett. 74, 156 (1999).

${ }^{278}$ R. Zoughi, Res. Nondestruct. Eval. 7, 71 (1995).

${ }^{279}$ L. Hao and J. C. Gallop, IEEE Trans. Appl. Supercond. 9, 1944 (1999).

${ }^{280}$ M. Tabib-Azar, R. Ciocan, G. Ponchak, and S. R. Leclair, Rev. Sci. Instrum. 70, 3387 (1999).

${ }^{281}$ M. Tabib-Azar and B. Sutapun, Rev. Sci. Instrum. 70, 3707 (1999).

${ }^{282}$ M. Tabib-Azar and S. R. LeClair, Sens. Actuators B 67, 112 (2000).

${ }^{283}$ M. Tabib-Azar, D. Akinwande, G. Ponchak, and S. R. LeClair, Rev. Sci. Instrum. 70, 3381 (1999).

${ }^{284}$ Karpov, R. W. Belcher, and J. H. Linn, Appl. Surf. Sci. 125, 332 (1998).

${ }^{285}$ M. J. Yoo, T. A. Fulton, H. F. Hess, R. L. Willet, L. N. Dunkleberger, R. J. Chichester, L. N. Pfeiffer, and K. W. West, Science 276, 579 (1997).

${ }^{286}$ M. Goldstein, G. Sölkner, and E. Gornik, Rev. Sci. Instrum. 64, 3009 (1993).

${ }^{287}$ J. B. Pendry, Phys. Rev. Lett. 85, 3966 (2000).

${ }^{288}$ R. A. Shelby, D. R. Smith, and S. Schultz, Science 292, 77 (2001).

${ }^{289}$ D. E. Steinhauer, C. P. Vlahacos, S. K. Dutta, B. J. Feenstra, F. C. Wellstood, and S. M. Anlage, Appl. Phys. Lett. 72, 861 (1998). 